\title{
The Golden Age of Cataclysmic Variables and Related Objects (A Very Personal Review)
}

\author{
Franco Giovannelli*† \\ INAF - Istituto di Astrofisica e Planetologia Spaziali, Via del Fosso del Cavaliere, 100, 00133 \\ Roma, Italy \\ E-mail: Eranco.giovannellidiaps.inaf.it
}

\begin{abstract}
In the last two years we assisted to a substantial improvement on the number of CVs discovered, but only few exciting results have been obtained. Thus, in this paper I cannot give astonishing news with respect to those discussed in the review paper published by Giovannelli \& SabauGraziati (2015a), but simply I will present a personal view about the route to be followed in the investigations on CVs. The exception has been the very exciting news about the discovery of a white dwarf pulsar in AR Sco (Buckley et al., 2017) presented by David Buckley in his talk (Bukley, 2017a, this workshop) and followed by a series of related talks by Beskrovnaya, Meintjes, Isakova, and Ikhsanov (2017, this workshop).
\end{abstract}

The Golden Age of Cataclysmic Variables and Related Objects IV

11-16 September, 2017

Palermo, Italy

* Speaker.

${ }^{\dagger}$ A footnote may follow. 


\section{Introduction}

The birth of the universe and its present status constitute the two banks of a river in which the life of the universe is slowly flowing. Undoubtedly the two banks are joined by a bridge that Giovannelli (2001a) nicknamed "The Bridge between the Big Bang and Biology" that constituted the title of the workshop held in Stromboli (Aeolian Archipelago, Sicily, Italy) in 1999. The big problem is how to cross this bridge, and the main question is: what are the experimental tools for understanding the pillars of this Bridge?

In order to cross this bridge, as always when we cross a bridge, we MUST advance slowly, step by step, with continuity, because everything is smoothly linked in the "magma" of the Universe, from the infinitely small to infinitely big, as sketched in Fig. 1 (adopted from Giovannelli \& SabauGraziati, 2017 after Rees, 1988 in Origins. The Darwin College lectures (1986) edited by Fabian, 1988).

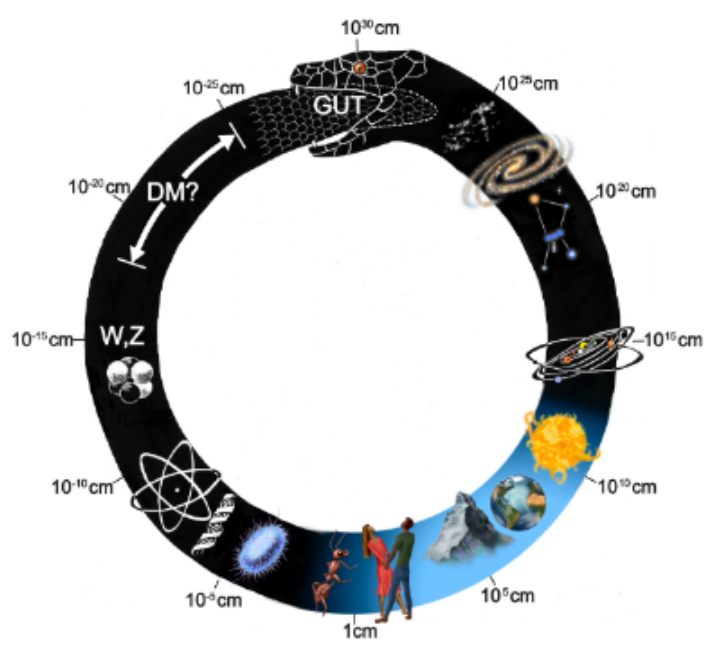

Figure 1: From the infinitely small to infinitely big (adopted from Giovannelli \& Sabau-Graziati, 2017 after Rees, 1988).

Indeed, if we look at the Fig. 2 (left panel) - where a section of the metabolic network of a "simple" bacterium is shown - we can note that each point (each chemical compound) is connected to any other point through the complexity of the network (Luisi \& Capra, 2014) exactly the same occurring in the "cosmic network" where each point is connected to any other point through the complexity of the network as shown in Fig. 2 (right panel) (https://it.wikipedia.org/wiki/Cosmologia del plasma). The large-scale structure of the Universe, as traced by the distribution of galaxies, is now being revealed by large-volume cosmological surveys. The structure is characterized by galaxies distributed along filaments, the filaments connecting in turn to form a percolating network. Shandarin, Habib \& Heitmann (2010) objective was to quantitatively specify the underlying mechanisms that drive the formation of the cosmic network. By combining percolation-based analyses with N-body simulations of gravitational structure formation, they elucidate how the network has its origin in the properties of the initial density field (nature) and how its contrast is then amplified by the nonlinear mapping induced by the gravitational instability (nurture). 

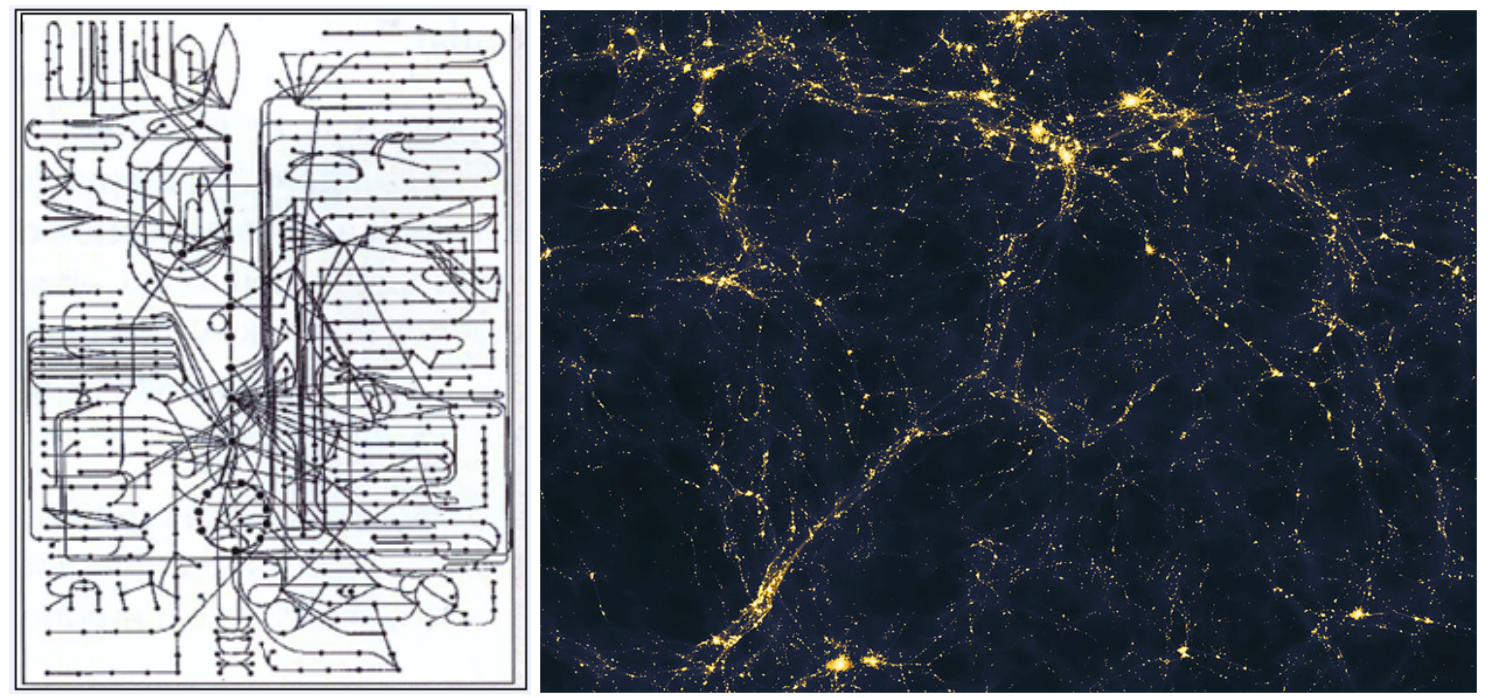

Figure 2: Left panel: Section of the metabolic network of a "simple" bacterium (Luisi \& Capra, 2014). Right panel: the "cosmic network" (https://it.wikipedia.org/wiki/Cosmologia del plasma) (adopted from Giovannelli \& Sabau-Graziati, 2017).

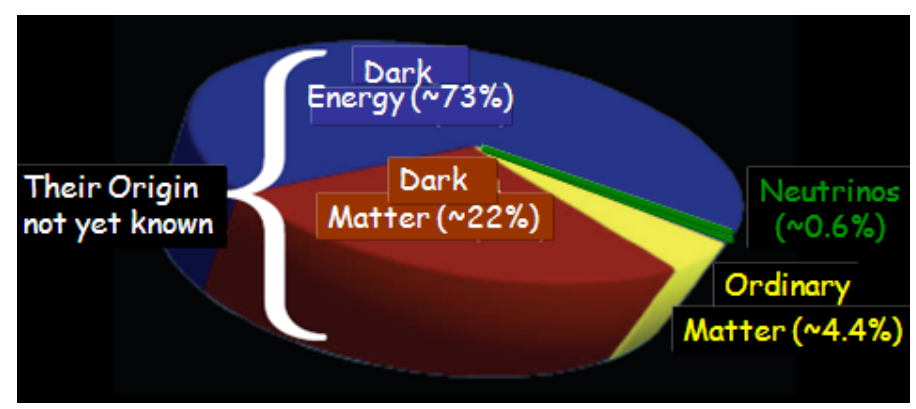

Figure 3: The cosmic budget.

As Albert Einstein affirmed, we can't solve problems by using the same kind of thinking we used when we created them. I can add something more, by using the wisdom: we can attach each kind of problem in a way as general as possible, and in any case it is necessary to go on without blinkers.

A fundamental question naturally arises: what is now the situation about our knowledge of the Universe? The answer is discouraging: we know a very small part of it, and not very well.

Indeed, Fig. 3 shows schematically such a situation. We can really discuss only on $\sim 5 \%$ of the content of the Universe. The remnant of $\sim 95 \%$ is almost completely unknown. However, the recent fundamental progress in gravitational astronomy could open an incredible source of information about this "unknown" content.

In this paper I will discuss the problem of Cataclysmic Variables and Related Objects, which constitute a small part of the "known" $\sim 5 \%$ of the content of the Universe. 


\section{Accretion Processes in Cosmic Sources}

Accretion is a universal phenomenon that takes place in the vast majority of astrophysical objects. The progress of ground-based and space-borne observational facilities has resulted in the great amount of information on various accreting astrophysical objects, collected within the last decades. The accretion is accompanied by the process of extensive energy release that takes place on the surface of an accreting object and in various gaseous envelopes, accretion disk, jets and other elements of the flow pattern. The results of observations inspired the intensive development of accretion theory, which, in turn, enabled us to study unique properties of accreting objects and physical conditions in the surrounding environment. One of the most interesting outcomes of this intensive study is the fact that accretion processes are, in a sense, self-similar on various spatial scales from planetary systems to galaxies.

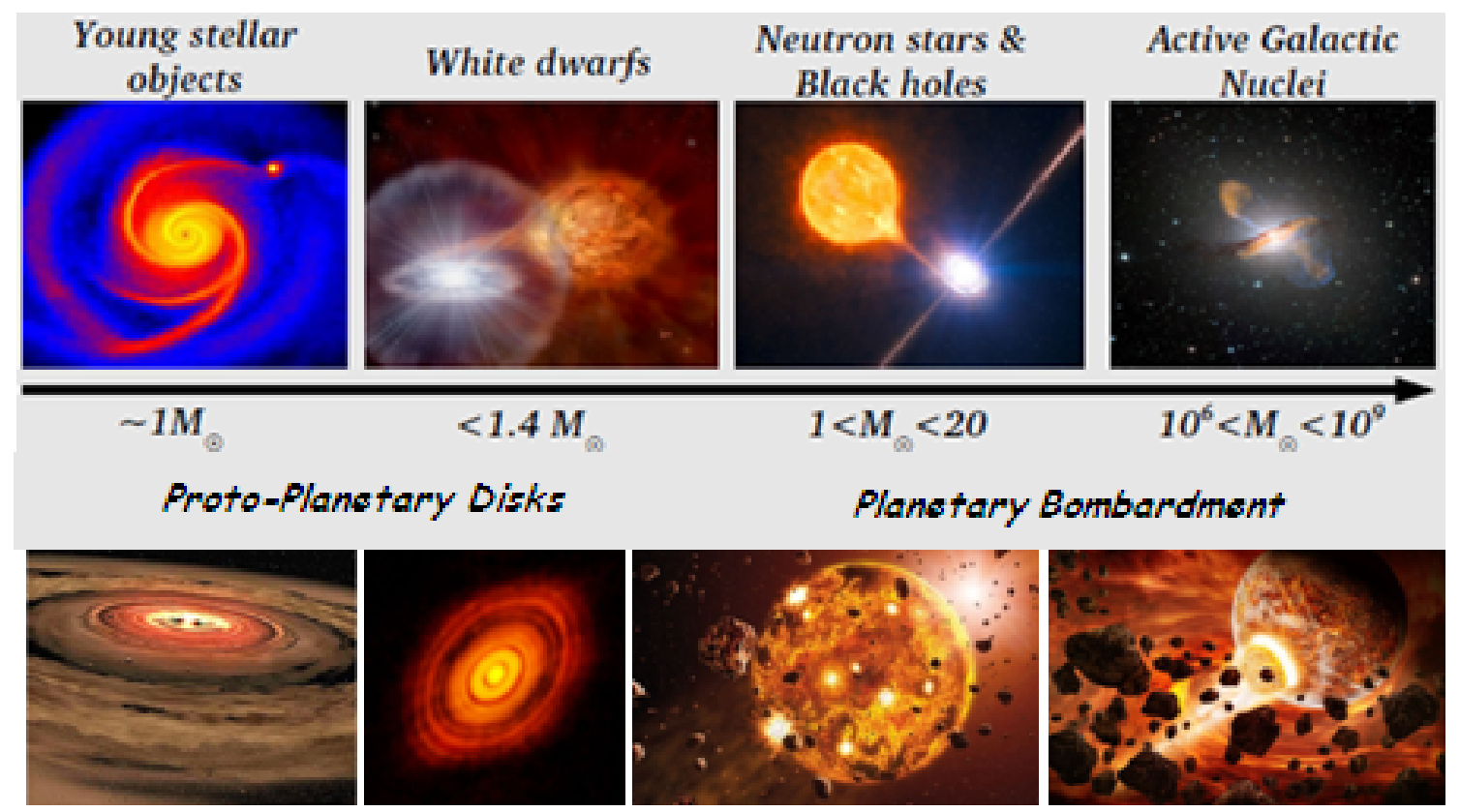

Figure 4: Accretion processes in different cosmic sources (adopted from Giovannelli \& Sabau-Graziati, 2016a, after Scaringi, 2015).

This fact gives us new opportunities to investigate objects that, by various reasons, are not available for direct study.

Cataclysmic variable stars are unique natural laboratories where one can conduct the detailed observational study of accretion processes and accretion disks.

Figure 4 shows a sketch of cosmic systems where accretion processes occur (Giovannelli \& Sabau-Graziati, 2016a, after Scaringi, 2015).

An international workshop on Accretion Processes in Cosmic Sources: Young Stellar Objects, Cataclysmic Variables (CVs) and Related Objects, X-ray Binary Systems, Active Galactic Nuclei was organized by me in collaboration with several colleagues of different international institutions, and took place in Saint Petersburg (Russian Federation) on September 2016. The proceedings discuss in details the physics of accretion processes in all the cosmic sources shown in Fig. 4 (Giovannelli \& Sabau-Graziati, 2016b). 


\section{About Cataclysmic Variables and Related Objects}

Historically, the classification of CVs was based on the optical outburst properties, by which one may distinguish four groups: (i) classical novae; (ii) recurrent novae; (iii) dwarf novae; (iv) nova-like objects.

This classification, however, is neither self-consistent nor adequate and it is much better to consider primarily the observed accretion behaviour (Smak, J.: 1985).

Figure 5 (left panel) shows the main characteristics of the visual light curves of classical novae (top panel) and of dwarf novae of the U Gem, Z Cam, and SU UMa types (lower three panels) (Ritter, 1992). In the right panel of Fig. 5 different kind of humans are reported. They show similarities with the light curves of CVs. Apparently they are different, but all of them belong to the same Human Species, like all the CVs are CVs.
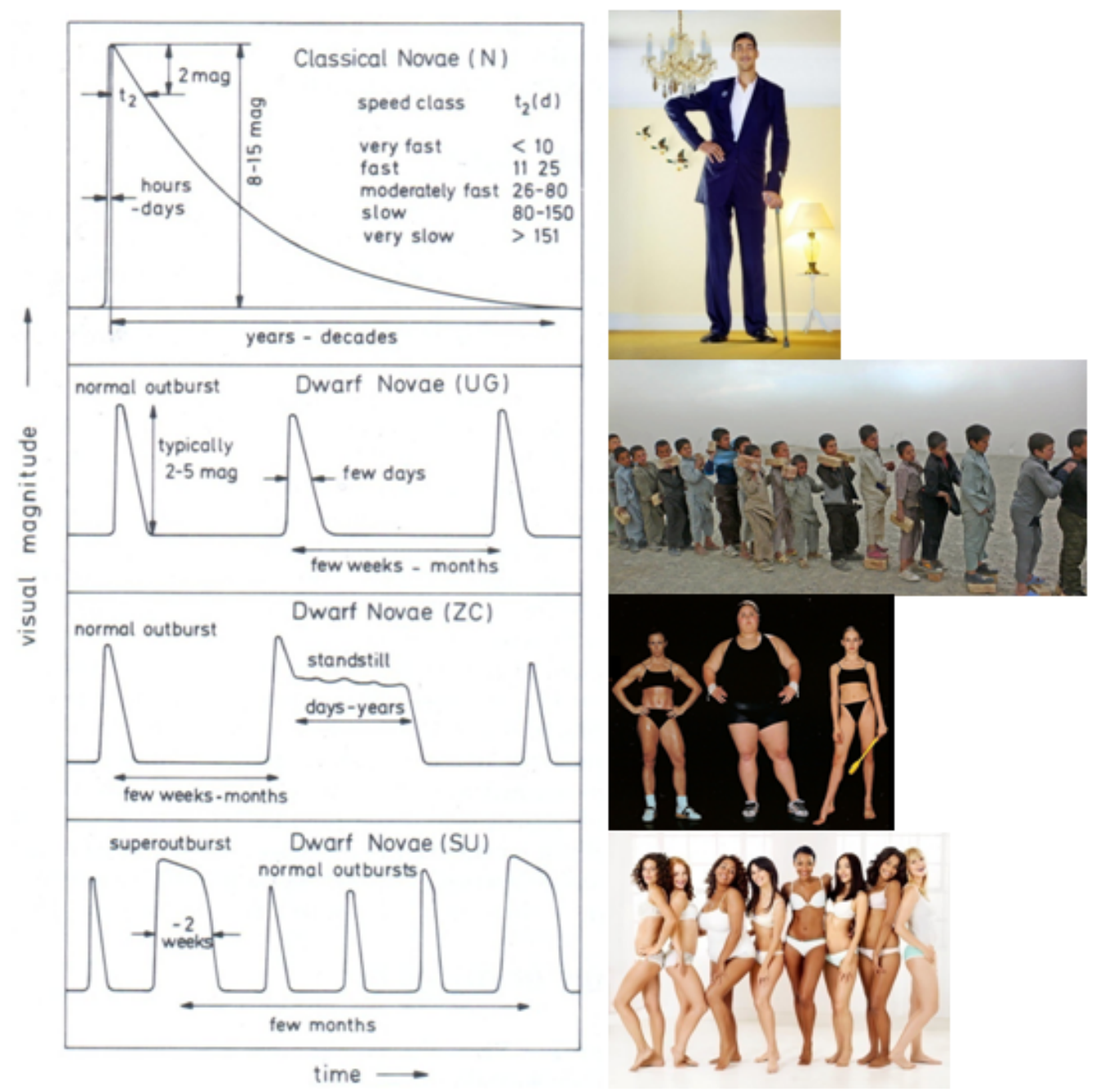

Figure 5: Left panel: the main characteristics of the visual light curves of CVs (Ritter, 1992). Right panel: different characteristics of humans.

Therefore, it is necessary to find a method as general as possible to describe the behavior of CVs. This can be obtained looking at the Accretion Behaviour and Magnetic Field. 


\subsection{Classification on the base of the magnetic field intensity}

The accretion structure depends on the magnetic field of white dwarf $\left(B_{1}\right)$ and on the transfer mass rate.

Following the popular classification, depending on $\mathrm{B}_{1}$ it is possible to classify $\mathrm{CV}$ s in three groups:

- Non Magnetic CVs (NMCVs): $\mathrm{B}_{1} \sim 10^{4}-10^{6} \mathrm{G}$;

- Intermediate Polars (IPs): $\mathrm{B}_{1} \sim 10^{6}-10^{7} \mathrm{G}$;

- Polars (MCVs): $\mathrm{B}_{1} \sim 10^{7}-10^{8} \mathrm{G}$.

Figure 6 shows such a classification. However we have a smooth continuity among the classes, as shown in fig. 7 (Giovannelli \& Sabau-Graziati, 2015a).
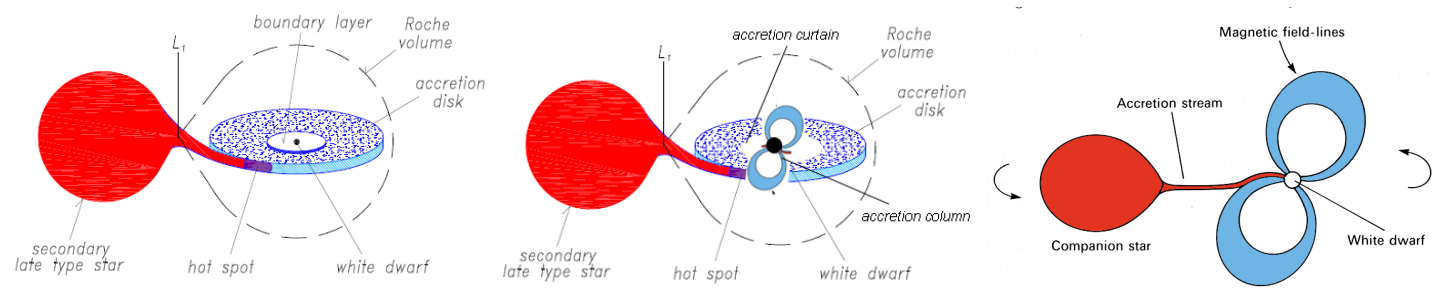

Figure 6: From left to right: sketch of NMCVs, IPs, and Polars.

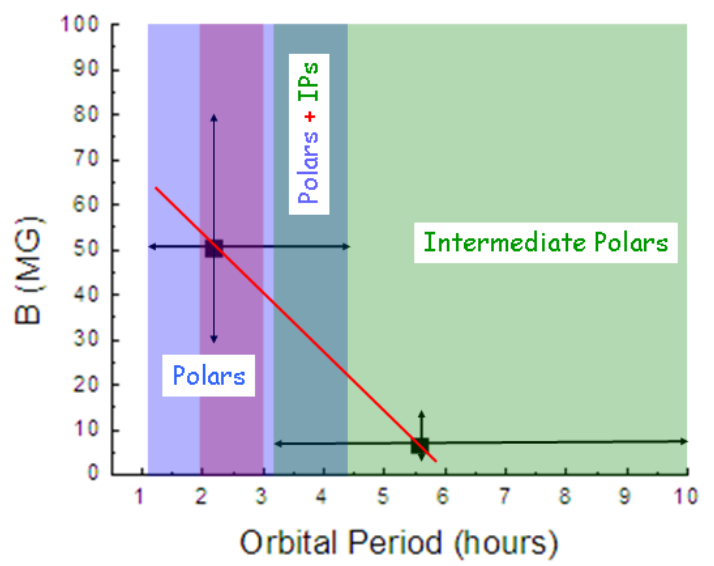

Figure 7: Magnetic field intensity versus orbital period for MCVs. Polars and IPs are contained in the light blue and light green rectangles, respectively. Violet rectangle indicates the so-called "period gap". Cyan-50 rectangle represents the intersection between the Polars and IPs (adopted from Giovannelli \& Sabau-Graziati, 2015a).

Indeed, taking into account the average values of magnetic field intensity and orbital periods for polars and IPs, and the minimum and maximum value for both parameters ( $\mathrm{B}$ and $\mathrm{P}_{\mathrm{orb}}$ ), it is possible to construct a very interesting plot (Fig. 7) that shows the evident continuity between the two classes of MCVs. Such a continuity has been noted by Schmidtobreick \& Tappert $(2014,2015)$ : 
CVs evolution is driven by angular momentum loss; as consequence $\mathrm{P}_{\text {orb }}$ decreases. All long $\mathrm{P}_{\text {orb }}$ CVs cross SW Sex regime before entering in the "period gap". Therefore SW Sex phenomenon is an evolutionary stage in the life of CVs (e.g. Rodriguez-Gil, 2003).

The nature in all its manifestations shows continuity. Then we have to abandon the "convenient method" of thinking everything in watertight compartments and to go toward a general model for compact accreting stars, like was done by Vladimir Lipunov and collaborators when they developed the "Scenario Machine".

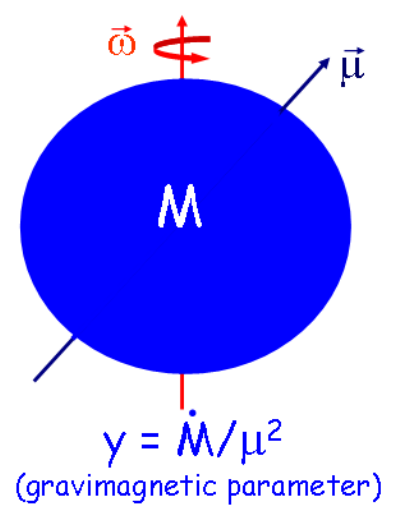

Figure 8: Gravimagnetic rotator: a body with mass $\mathrm{M}$, having a magnetic moment $\vec{\mu}$, rotating with rotational velocity $\vec{\omega}$ (adopted from Giovannelli, 2016). The parameter $\mathrm{y}=\dot{\mathrm{M}} / \mu^{2}$ is called gravimagnetic parameter (Lipunov, 1987; Lipunov \& Postnov, 1988).

Starting from the trivial definition of X-ray Binary Systems (XRBs): they are binary systems emitting X-rays, a natural question arises. Are these systems governed by few physical parameters independent of their nature? The answer is positive. Indeed, High Mass XRBs (HMXRBs), Low Mass XRBs (LMXRBs), Anomalous X-ray Pulsars (AXPs), and Cataclysmic Variables (CVs) can be considered as gravimagnetic rotators: a body with mass $\mathrm{M}$, having a magnetic moment $\vec{\mu}$, rotating with rotational velocity $\vec{\omega}$, being the two axis not necessarily coincident, as sketched in Fig. 8 (Giovannelli, 2016). Introducing a physical parameter, $y=\dot{M} / \mu^{2}$, named gravimagnetic parameter, all the gravimagnetic rotators are contained in a plane $\log \mathrm{P}_{\text {spin }} \mathrm{vs} \log \mathrm{y}$ (Lipunov, 1987; Lipunov \& Postnov, 1988).

The Scenario Machine (Monte Carlo simulations of binary evolution) permits to build up the complete picture of all possible evolutionary stages of binaries in the Galaxy. The basic evolution equation (3.1) used for 500,000 systems containing magnetized stars provided the results contained in the plane $\log \mathrm{P}_{\text {spin }}-\log \mathrm{y}$, reported in the upper panel of Fig. 9. $\mathrm{P}_{\text {spin }}$ is expressed in seconds and the gravimagnetic parameter is expressed in unit of $10^{-42} \mathrm{~g} \mathrm{~s}^{-1} \mathrm{G}^{-2} \mathrm{~cm}^{-6}$. The symbols used for the different types of binaries are explained in the lower panel of Fig. 9. The definition of the characteristic radii can be found in the paper by Lipunov (1987). Observational examples of various types of rotators are reported in Fig. 10 (Lipunov, 1987).

$$
\frac{\mathrm{dI} \omega}{\mathrm{dt}}=\dot{\mathrm{M}} K_{\mathrm{su}}-\frac{\kappa_{\mathrm{t}} \mu^{2}}{\mathrm{R}_{\mathrm{t}}^{3}}
$$

where: 


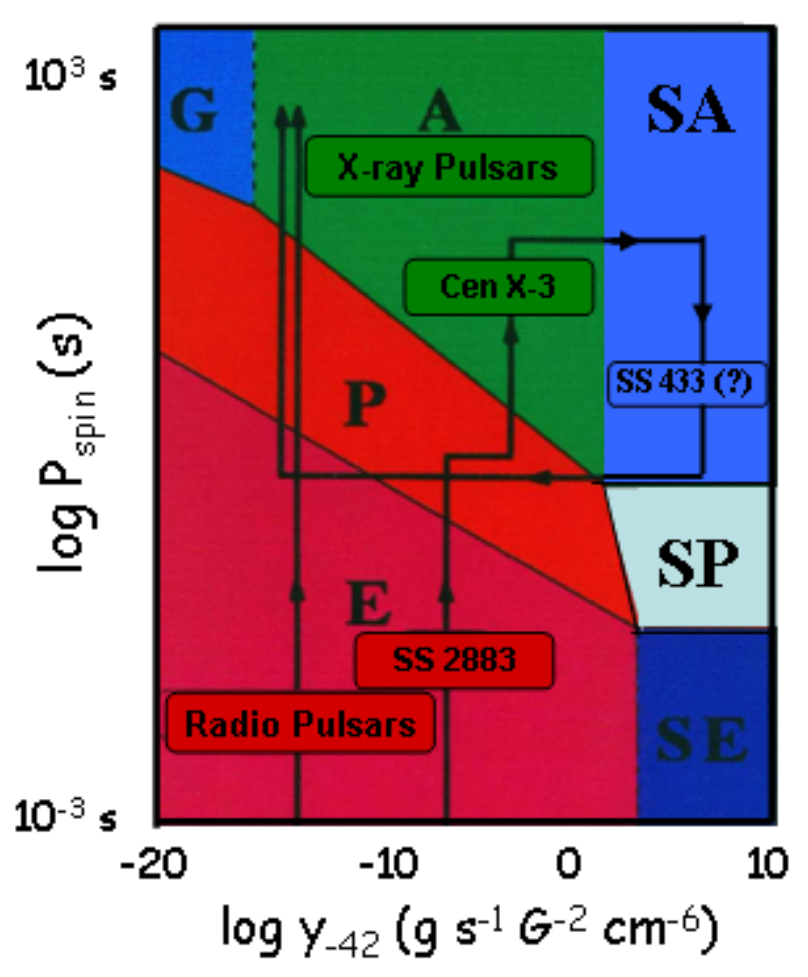

The rotator classification

\begin{tabular}{|c|c|c|}
\hline Designation & Name & Physical sense \\
\hline $\mathrm{E}$ & Ejector & $R_{s t}>\max \left\{R_{G}, R_{l}\right\}$ \\
\hline $\mathbf{P}$ & Propeller & $R_{c}<R_{s t} \leq \max \left\{R_{G}, R_{t}\right\}$ \\
\hline A & Accretor & $R_{s t} \leq R_{G}$ and $R_{s t} \leq R_{c} \quad \dot{M}_{c} \leq \dot{M}_{c r}$ \\
\hline G & Georotator & $R_{G}<R_{s t} \leq R_{c}$ \\
\hline M & Magnetor & $R_{s t}>a$ and $R_{c}>a$ \\
\hline SE & Superejector & $R_{s t}>R_{l}$ \\
\hline SP & Superpropeller & $R_{c}<R_{s t} \leq R_{l}$ \\
\hline SA & Superaccretor & $R_{s t} \leq R_{c}$ and $R_{s t} \leq R_{G}$ \\
\hline
\end{tabular}

Figure 9: Upper panel: distribution of magnetic rotators in the plane "Spin Period" versus "Gavimagnetic Parameter" (adopted from Giovannelli, 2016 after Lipunov, 1995); lower panel: classification of rotators (Lipunov, 1987).

$\mathrm{K}_{\mathrm{su}}=$ specific angular momentum applied by the accretion matter to the rotator;

$\mathrm{K}_{\mathrm{su}}=\sqrt{\mathrm{GM}_{\mathrm{x}} \mathrm{R}_{\mathrm{d}}}$ for Keplerian disk accretion;

$\mathrm{K}_{\mathrm{su}}=\eta_{\mathrm{t}} \Omega R_{\mathrm{g}}^{2}$ for wind accretion in a binary;

$\mathrm{K}_{\mathrm{su}} \sim 0$ for a single magnetic rotator;

$R_{d}=$ radius of the inner disk edge;

$\Omega=$ rotational frequency of the binary system;

$\eta_{\mathrm{t}}=1 / 4 \quad$ (Illarionov \& Sunyaev, 1975);

$\kappa_{\mathrm{t}}=$ dimensionless factor;

$\mathrm{R}_{\mathrm{t}}=$ characteristic radius;

$\dot{\mathrm{M}}=$ accretion rate in different regimes. 


\begin{tabular}{|c|c|c|c|}
\hline Type of rotator & Designation & $\begin{array}{l}\text { The clearly confirmed } \\
\text { observational example }\end{array}$ & Model assumptions \\
\hline Ejector & $\mathrm{E}$ & Radiopulsars & $\begin{array}{l}\text { LSI }+61^{\circ} 303, \text { Cyg X-3, } \\
\text { BL Lac objects, }\end{array}$ \\
\hline Propeller & $\mathrm{P}$ & - & $\begin{array}{l}\text { Transient X-ray sources, } \\
\gamma \text {-bursts, some cataclysmic } \\
\text { variables (dwarf novae), } \\
\text { magnetic Ap-stars }\end{array}$ \\
\hline Accretor & A & $\begin{array}{l}\text { X-ray pulsars, } X \text {-ray } \\
\text { bursters, cataclysmic } \\
\text { variables with white } \\
\text { dwarfs, novae, intermediate } \\
\text { polars }\end{array}$ & - \\
\hline Superejector & SE & - & SS 433, AGN, QSO \\
\hline Superpropeller & SP & - & - \\
\hline Superaccretor & SA & - & SS 433 \\
\hline Georotator & G & - & - \\
\hline Magnetor & M & Polars & - \\
\hline
\end{tabular}

Figure 10: Observational examples of rotators (Lipunov, 1987).

\subsection{High Magnetic Field White Dwarfs (HMFWDs)}

Magnetic fields are observed in main sequence stars and their white dwarf and neutron star progeny. The fields in these three groups of stars are likely to be linked via stellar evolution. However, stellar evolution calculations that include magnetic fields and rotation in a self consistent manner are only now beginning to be carried out, and then only with simplifying assumptions. Some insights into the nature of magnetism of stars in these three groups can be obtained by a comparative study of their group properties (e.g. Ferrario \& Wickramasinghe, 2007).

While there is strong evidence that the magnetic fields in late type stars are dynamo generated, it is likely that the magnetic fields of stars on the upper main sequence are of fossil origin, perhaps dating back to the time of star formation.

We have the following approximate correspondences under flux conservation:

$$
\begin{aligned}
& \mathrm{B}_{\mathrm{B}-\mathrm{F}} \sim 300-30000 \mathrm{G} \Longrightarrow \mathrm{B}_{\mathrm{HFMWD}} \sim 10^{6}-10^{9} \mathrm{G} \\
& \mathrm{B}_{\mathrm{O}-\mathrm{B}} \sim ? ?-2000 \mathrm{G} \Longrightarrow \mathrm{B}_{\mathrm{NS}} \sim 10^{11}-10^{15} \mathrm{G}
\end{aligned}
$$

The lower field limit is still unknown for early B to O-type stars. A strong case can therefore be made for a fossil origin of fields at least at the high field end of the field distribution (Ferrario \& Wickramasinghe, 2007).

The rotational periods of WDs and NSs are scaling as $\mathrm{P}_{\mathrm{NS}} / \mathrm{P}_{\mathrm{WD}} \approx\left(\mathrm{R}_{\mathrm{NS}} / \mathrm{R}_{\mathrm{WD}}\right)^{2}$.

There are two possible origin of strong magnetic fields in WDs:

- The fossil field model (no stellar evolution models show how strong fossil magnetic flux can survive through the various stages of stellar evolution through to the WD phase).

- The merging star model (dynamo model of Wickramasinghe, Tout \& Ferrario, 2014): similar maximum magnetic fluxes may be expected for physical reasons if the fields are generated from differential rotation caused by merging).

In the fossil scenario, the magnetic field strength $B$ scales with the radius of the star $R_{\star}$ as $B \propto$ $\mathrm{R}_{\star}{ }^{-2}$ (Ferrario, Melatos \& Zrake, 2015). 
Figure 11 shows the distribution of rotation periods for magnetic white dwarfs (MWDs) (left panel) and neutron stars (NSs) (right panel) (adapted from Ferrario \& Wickramasinghe, 2005). Both diagrams have the same trend, except the scale factors of rotational periods $\mathrm{P}_{\text {rot }}$ (minutes for WDs, seconds for NSs) and magnetic field intensity ( $\mathrm{B} \sim 10^{7}-10^{9}$ for WDs and $\sim 10^{13}-10^{15} \mathrm{G}$ for NSs).

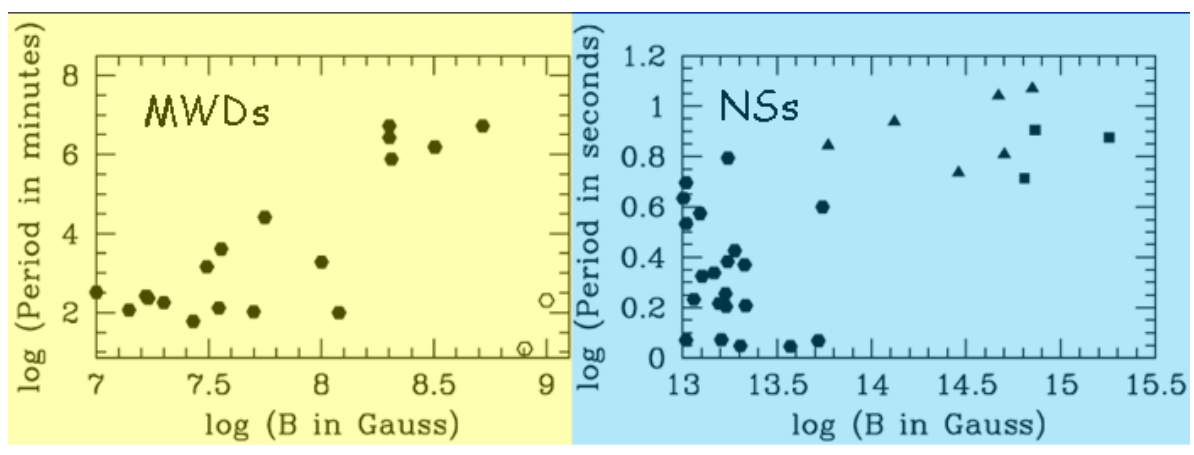

Figure 11: The distribution of rotation periods for magnetic white dwarfs (MWDs) (left panel) and neutron stars (NSs) (right panel) (adapted from Ferrario \& Wickramasinghe, 2005).

We still need to construct: (i) more realistic model atmospheres that allow for the presence of magnetic fields; (ii) stellar evolution tracks of intermediate mass stars that take into consideration both fossil and dynamo generated fields. Such calculations may be able to tell us whether all WDs are magnetic at some level.

The origin of fields in highly magnetic WDs is currently being debated. Although the newly proposed scenario that all high field MWDs (single and in binaries) are the result of close binary evolution and mergers is gaining momentum, the fossil field hypothesis cannot be totally dismissed (Ferrario, de Martino \& Gänsicke, 2015).

It is interesting to note in Fig. 12 the distribution of B for MCVs and MWDs (adapted from Ferrario, de Martino \& Gänsicke, 2015).

In MCVs there is an interesting relationship between the magnetic field strength and orbital period of the systems, as reported in Fig. 13 where the polars and IPs are separated by the blue line that marks the synchronization between orbital and spin periods of the cataclysmic systems (Giovannelli \& Sabau-Graziati, 2015a after Ferrario, de Martino \& Gänsicke, 2015).

Also the system AM CVn $\equiv$ HZ 29 with orbital period of 17.5 minutes, discovered by Smak (1967) was later recognized as a CV by Patterson (1992) and it constitutes the prototype of AM CVn stars - ultra-compact binaries - formed by a primary star WD, and a secondary degenerate or semi-degenerate star: e.g. white dwarf - white dwarf binaries $\left(\mathrm{P}_{\text {orb }}<80\right.$ minutes $)$. White dwarf primaries and main sequence secondaries have binary orbital periods greater than 80 minutes.

Levitan (2013) in his PhD thesis about "AM CVn Systems with Palomar Transient Factory" updates the number of AM CVn stars. Figure 14 shows the number of CVs versus orbital period. It is clearly evident that $50 \%$ of CVs lie over the "period gap", $39 \%$ of CVs lie below the "period gap", and $11 \%$ within the "period gap" (indicated with the light blue rectangle). The place where SW Sex systems lie (indicated with the light red rectangle) is partially in the "period gap". This 


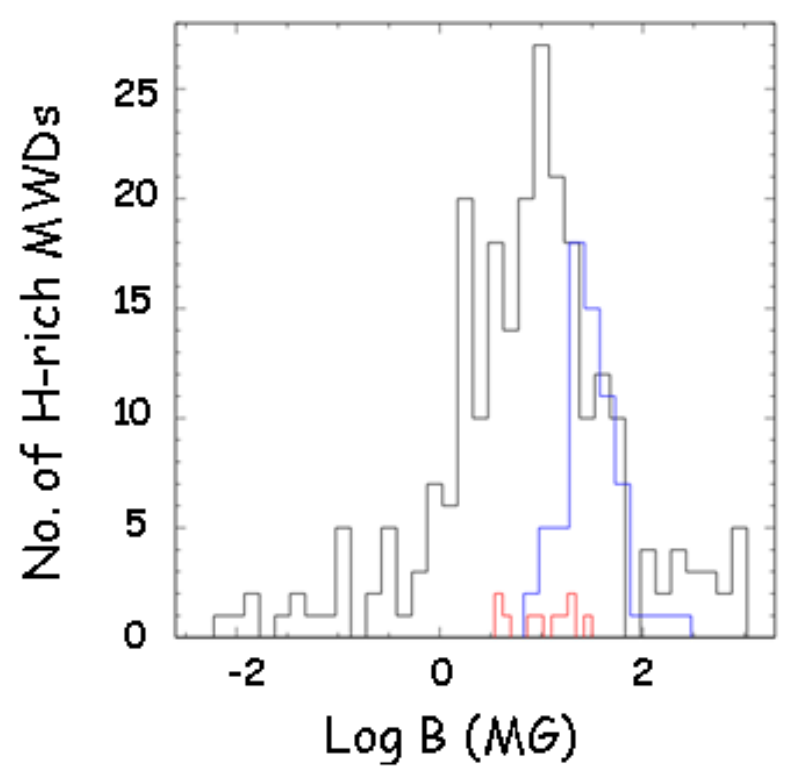

Figure 12: Distribution of magnetic field strength in polars (blue line), and IPs (red line) compared to that of single magnetic WDs (black line) (adapted from Ferrario, de Martino \& Gänsicke, 2015).

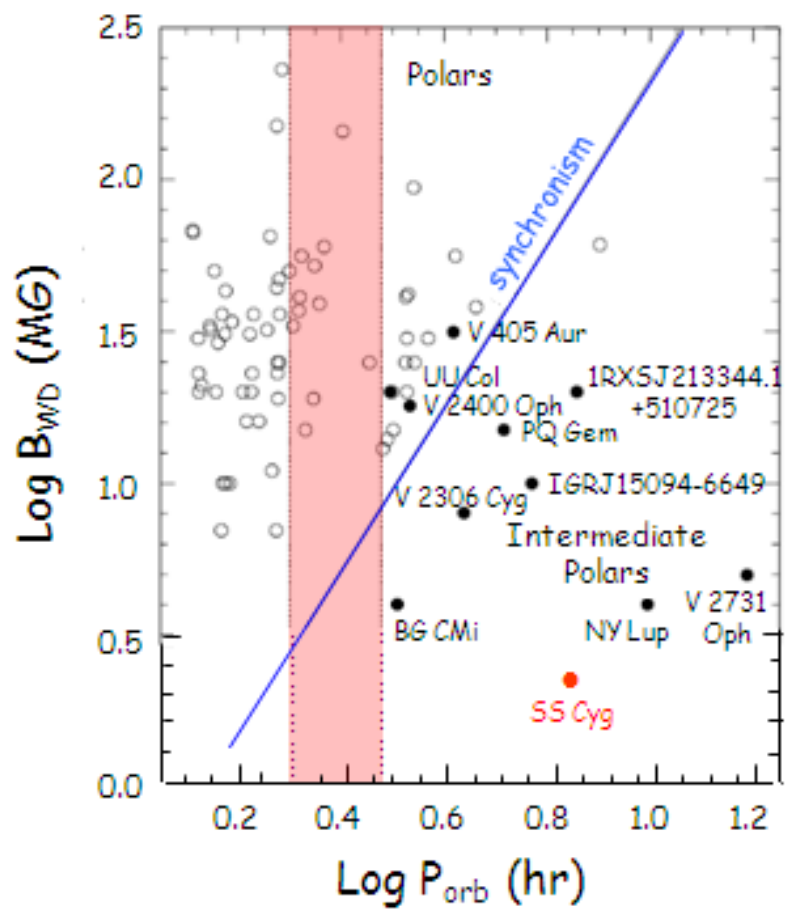

Figure 13: Magnetic field strength versus orbital period in MCVs. Polars and IPs are separated by the blue line that marks the border between systems with orbital period synchronized with the spin period (polars) and those without synchronization (IPs). Light red rectangle marks the so-called "period gap" (adopted from Giovannelli \& Sabau-Graziati, 2015a after Ferrario, de Martino \& Gänsicke, 2015). 


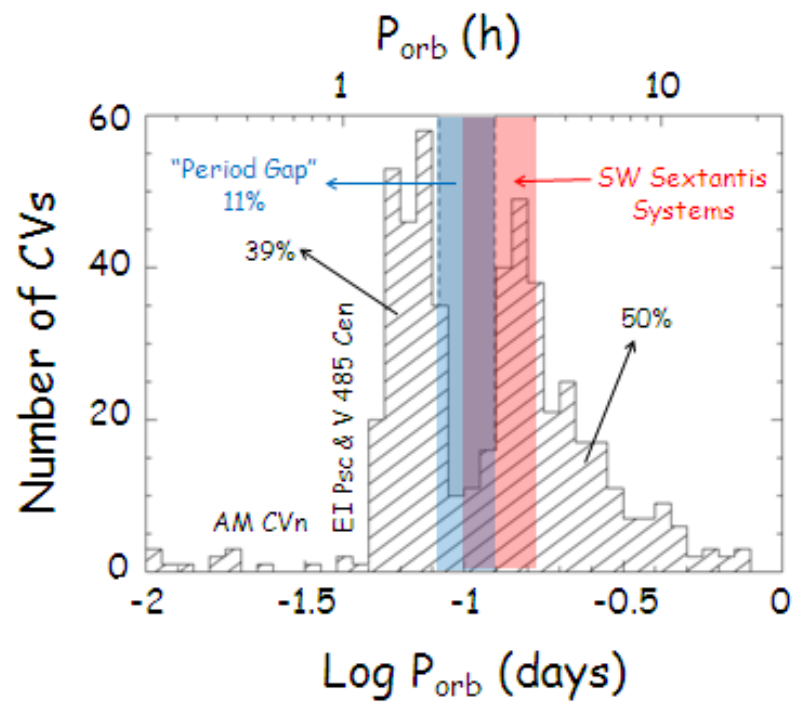

Figure 14: Number of CVs versus $P_{\text {orb }}$. Light blue and red rectangles mark the so-called "period gap" and the range in whicg SW Sex systems lie (adopted from Giovannelli \& Sabau-Graziati, 2015a after Gänsicke, 2005, and Levitan, 2013).

demonstrates that the "period gap" does not exist. It appeared in the past like a gap because of lack of measurements and relatively low number of systems within the gap.

In my opinion is extremely important to remark once more that there is continuity among the "different classes" of CVs. This is coming from the pathway of evolution of CVs that is driven by angular momentum $(\mathrm{J})$ loss. Orbital period $\left(\mathrm{P}_{\text {orb }}\right)$ decreases. All long $\mathrm{P}_{\text {orb }} \mathrm{CV}$ s cross SW Sex regime before entering in the "Period Gap", where SW Sex systems lie. Thus, SW Sex phenomenon is an evolutionary stage in the life of CVs (Rodriguez-Gil, 2003; Schmidtobreick, 2013).

An exhaustive discussion about the state of art of the possible evolution of CVs including AM CVn systems is reported in the review paper by Giovannelli \& Sabau-Graziati (2015a) and references therein.

\subsection{How to search B indirectly in MCVs}

It is convenient to remind how the magnetic field intensity can be measured. A detailed discussion about the field determination in isolated magnetic WDs and in WDs in binary systems is reported in the review paper by Ferrario, de Martino \& Gänsicke (2015). Direct measurements of the WD magnetic field strength in the high field magnetic CVs, the polars, can be obtained either (i) through Zeeman splitting of the photospheric hydrogen absorptions lines when these systems enter low accretion states or (ii) through the modeling of cyclotron emission features that characterizes the optical to IR spectra during intermediate and high accretion states (see Wickramasinghe \& Ferrario, 2000) or (iii) via the study of Zeeman features arising from the halo of matter surrounding the accretion shock.

In my opinion it is important to remark an interesting indirect method for evaluating the magnetic field intensity in MCVs as discussed by Giovannelli \& Sabau-Graziati (2012a) in the case of SS Cyg whose nature (non magnetic or IP) is largely disputed. From the fluxes of UV emission 
lines of SS Cyg, placed at distance $d=166 \pm 7$ pc (Harrison et al., 1999), Giovannelli \& SabauGraziati (2012a) - by using the IUE measurements obtained by Gaudenzi et al. (1986) - derived the luminosity of C II and C IV: $\mathrm{L}_{\mathrm{CII}} \simeq 7.8 \times 10^{30} \mathrm{erg} \mathrm{s}^{-1}$ and $\mathrm{L}_{\mathrm{CIV}} \simeq 6.2 \times 10^{31} \mathrm{erg} \mathrm{s}^{-1}$. Using these values of luminosity and the extrapolation of the line best fitting the emission line luminosity of $\mathrm{C}$ II and C IV versus B (Howell et al., 1999), the magnetic field intensity of SS Cyg is $\mathrm{B}_{\mathrm{CII}}=2.0_{-0.4}^{+0.5}$ $\mathrm{MG}$, and $\mathrm{B}_{\mathrm{CIV}}=1.1_{-0.6}^{+0.3} \mathrm{MG}$, as shown in Fig. 15, left and right panels, respectively.
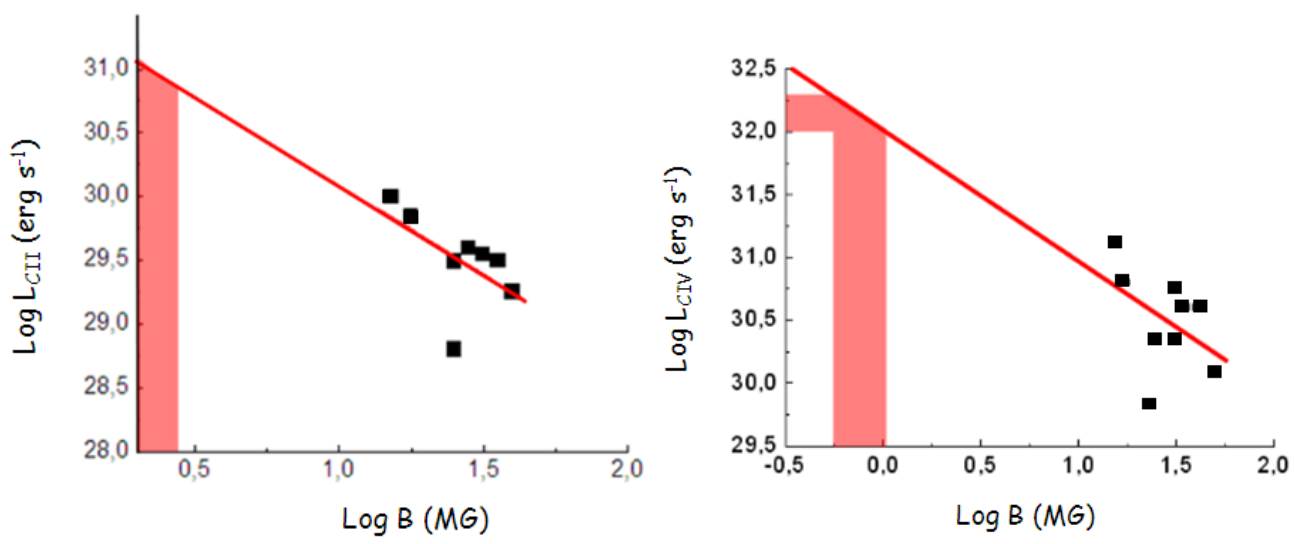

Figure 15: Observed emission fluxes converted to luminosity for magnetic CVs (after Howell et al., 1999) are indicated with black square. Left panel: C II luminosity versus B; right panel: C IV luminosity versus B. SS Cyg positions are indicated with red areas (adopted from Giovannelli \& Sabau-Graziati, 2012a).

Then a reasonable value of the white dwarf magnetic field in SS Cyg is $\mathrm{B}=1.6 \pm 0.7 \mathrm{MG}$ This value is in complete agreement with the evaluation made by Fabbiano et al. (1981) $(\mathrm{B} \leq 1.9$ MG) by using simultaneous X-ray, UV, and optical data.

Many other circumstantial proofs in favor of the IP nature of SS Cyg have been discussed by Giovannelli \& Sabau-Graziati (2012a). One of the most important proof is coming from the paper by Körding et al. (2008). They detected a radio jet from SS Cyg. The hardness intensity diagram shows an analogy between the XRBs (the BH GX 339-4, and the NS Aql X-1) and SS Cyg. Moreover there is a radio flare simultaneous with the optical outburst of SS Cyg. During the 1.1-mJy "flare" they found upper limits for the linear polarization and circular polarization of $3.2 \pm 2.7 \%$ and $-3.2 \pm 2.7 \%$, respectively.

It is hard to explain these results without invoking the presence of a magnetic field ( $\mathrm{B} \approx 2$ MG) in the white dwarf of SS Cyg system.

INTEGRAL/IBIS and SWIFT/XRT observations have shown that a conspicuous number of CVs have a strong hard X-ray emission (Landi et al., 2009; Scaringi et al., 2010). In their published sample of $23 \mathrm{CVs}, 22$ are classified as magnetic IPs and only one (SS Cyg) as NMCV, meanwhile all its characteristics are practically equal to those of the other 22 objects. This is a strong circumstantial proof in favor of the magnetic nature of SS Cyg. The experimental evidence that SS Cyg emits in the hard X-ray energy range is, in my opinion, the conclusive evidence about its magnetic nature.

Moreover, a simple question arises: "why all the CVs detected by the INTEGRAL observatory are magnetic (IPs) and SS Cyg non-magnetic? If so, why the same INTEGRAL observatory did 
not detect any other DN?".

The hard X-ray emission detected in those CVs is possible only if a sufficiently high magnetic field is present in those systems. Only accretion onto magnetic poles justify the hard X-ray emission in quiescence.

Probably, the "mistake" about the nature of SS Cyg born after the publication of a paper in Nature by Bath \& van Paradijs (1983) where SS Cyg was classified as DN on the basis of optical behaviour, typical of DNe. This paper originated a bandwagon effect in the literature (see Michael Friedjung's comment in the first historical Frascati Workshop 1984: Giovannelli, 1985) that "obliged" almost all the subsequent authors to start the papers saying that SS Cyg is a DN (NMCVs), without paying attention to other possibilities well documented in the so-called second class literature.

Therefore, my suggestion is to reconsider the problem about the nature of SS Cyg without any a priori bias.

There is an additive interesting proof about the possibility that SS Cyg is a MCV. Indeed if the ratio between the fluxes of UV lines is used, it is possible to see that SS Cyg lies in a position which is compatible with the best fit for both the IPs and polars. The UV lines used are NV, SiIV and CIV. The logarithm of ratio of the fluxes of NV and SiIV and NV and CIV gives the plots of Fig. 16. In the left and right panels are reported the IPs and polars, respectiveely. The position of SS Cyg is marked in red (adopted from Gaudenzi et al., 2002).
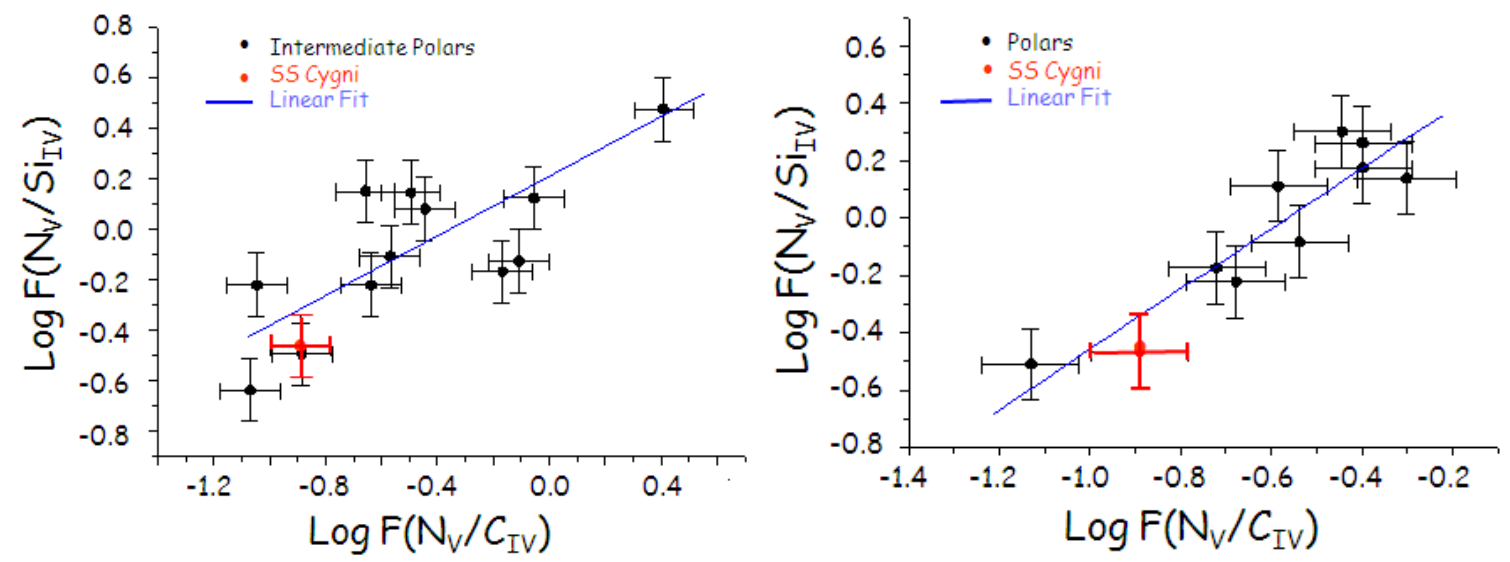

Figure 16: Relationship between the ratio of the fluxes of UV lines NV/SiIV versus NV/CIV in logarithmic scales. Left panel refers to IPs; right panels refers to polars. The position of SS Cyg is marked in red in both panels (adopted from Gaudenzi et al., 2002).

My opinion is that a more appropriate investigation of the class of the so-called IPCVs is necessary.

However, all the discussion about CVs would teach a lesson: it is mandatory to observe CVs for long time and possibly with simultaneous observations across the electromagnetic spectrum, from radio to gamma rays in order to follow at least a whole period of the binary system between two successive outbursts. This is, of course, possible only for systems like dwarf novae where the almost periodical outbursts occur in time scales of weeks-months. Networks of robotic telescopes can help in this matter (Giovannelli \& Sabau-Graziati, 2012b) 


\subsection{Doppler tomography of accretion disks}

The fast Fourier Transform (FFT) in one, two, or three dimensions is an efficient algorithm well known for decades (see Cooley \& Tukey, 1965) as well as a traditional component of any serious numerical library. Three-dimensional (3D) FFT happens to be one of the most compute- and communication-intensive components in applications from a range of fields (for example, in turbulence, molecular dynamics, 3D tomography, and astrophysics), and for this reason there are many excellent parallel implementations in existence today. Pekurovsky (2012) introduced a popular software package called P3DFFT which implements FFT in three dimensions in a highly efficient and scalable way. This paper discussed P3DFFT implementation and performance in a way that helps guide the user in making optimal choices for parameters of their runs.

Horne (1985) and Marsh \& Horne (1988) developed Doppler tomography as a technique aimed at constructing a two-dimensional velocity image (tomogram) of the accretion disks of CVs using an emission line in its spectra sampled at a number of orbital phases. Doppler tomography has revolutionized the interpretation of orbitally phase-resolved spectroscopic observations of interacting binary systems. It has become a valuable tool for resolving the distribution of line emission in CVs and other binary systems. For instance Marsh (2001) in his review discussed the results of Doppler tomography applied to CVs. Outstanding successes to that date are the discovery of two-arm spiral shocks in cataclysmic variable accretion disks and the probing of the stream/magnetospheric interaction in magnetic cataclysmic variable stars. Doppler tomography has also told us much about the stream/disk interaction in non-magnetic systems and the irradiation of the secondary star in all systems.

Zhilkin \& Bisikalo (2009) described a 3D numerical code designed for simulations of magnetohydrodynamical (MHD) flows in semidetached binary systems. They found that even in case of a moderate magnetic field $\left(B=10^{5} \mathrm{G}\right)$ the flow structure in the vicinity of the accretor of a $\mathrm{CV}$ was drastically changed and the funnel flows were formed in the system.

Bisikalo \& Zhilkin (2012) discussed the Flow Structure in Magnetic CVs by studying the physical processes which go on in magnetic CVs with the mass transfer between the components. Using results of 3D MHD simulations, they investigated variations of the main characteristics of accretion disks depending on the value of the magnetic induction on the surface of the accreting star. Zhilkin, Bisikalo \& Mason (2012) discussed on "Full 3D MHD calculations of accretion flow structure in magnetic cataclysmic variables with strong, complex magnetic fields".

Bisikalo et al. (2008) discussed Dopppler tomography of the CV SS Cygni in quiescence. Boneva et al. (2009) discussed the Doppler mapping in the case of SS Cygni in outburst and finally Kononov et al. (2012) used Doppler tomography for studying the pre-outburst accretion disk of SS Cygni.

Zhilkin \& Bisikalo (2011) presented a 3D numerical model to simulate plasma flows in close binary systems with accretors having strong proper magnetic fields. The model is based on an assumption that plasma dynamics is determined by a slow average flow against a background of propagating MHD waves. Equations describing the slow plasma motion are obtained as a result of the corresponding averaging procedure. The model takes into account the magnetic diffusivity which is due to the current dissipation in turbulent vortexes, magnetic buoyancy and wave MHD turbulence. They presented results of the simulations for MHD flows in a polar-like binary system. 
Results of the simulations show that in semi-detached binary systems with strong magnetic fields $\left(B=10^{7} \mathrm{G}\right)$ the flow structure can differ sufficiently from that of systems with moderate magnetic fields $\left(B=10^{5} \mathrm{G}\right)$, Accretion disks in such magnetic systems systems $\left(B=10^{7} \mathrm{G}\right)$ are not formed and the flow is collimated into the accretion stream starting from the inner Lagrange point $L_{1}$ and finishing on the magnetic poles of the accretor. This flow structure corresponds to the case of polars.

Kotze, Potter \& McBride $(2015,2016)$ explored inside-out Doppler tomography for nonmagnetic and magnetic cataclysmic variables, respectively. The code they used is available at http://www.saao.ac.za/ ejk/doptomog/main.html.

Doppler tomography has been applied also to fusion plasmas giving an image of the fastion velocity distribution function in the tokamak ASDEX Upgrade (Salewski et al., 2015). It is interesting to note that Bisikalo et al. (2016) presented a method that can be used to recover the spectrum of turbulence from observations of optically thin emission lines formed in astrophysical disks. Within this method, they analyze how line intensity fluctuations depend on the angular resolution of the instrument, used for the observations. The method allows us to restore the slope of the power spectrum of velocity turbulent pulsations and estimate the upper boundary of the turbulence scale.

Doppler tomography is very useful also for analyzing the asymmetric MHD outflows/jets from accreting T Tauri stars, and even in planetary science, as discussed by Giovannelli \& Sabau-Graziati (2016a, and the references therein).

\section{The tools for exploring the Universe}

The Universe manifests not only through electromagnetic radiation but also through astroparticles, including neutrinos, and recently through gravitational waves. Therefore, multifrequency observations, possibly simultaneous, are fundamental in photonic astrophysics, particle astrophysics, and gravitational wave astrophysics.

Thus it is evident the necessity of many kinds of experiments in different frequency regions. Upstream of this we have to distinguish two great classes of experiments: big experiments and small experiments.

\subsection{Big experiments}

Several examples of big experiments are:

a) The Astrometry Mission GAIA (Rix \& Bovy, 2013) is making the largest, most precise threedimensional map of our Galaxy by surveying more than a thousand million stars. GAIA will monitor each of its target stars about 70 times over a five-year period. It will precisely chart their positions, distances, movements, and changes in brightness. It is expected to discover hundreds of thousands of new celestial objects, such as extra-solar planets and brown dwarfs, and observe hundreds of thousands of asteroids within our own Solar System. The mission will also study about 500,000 distant quasars and will provide stringent new tests of Albert Einstein's General Theory of Relativity. 
GAIA is providing strong impact on stellar evolution and in calibrating the energy released by cosmic sources. In particular GAIA will be fundamental in giving the distances of the many CVs discovered and therefore their luminosity for the benefit of a correct evolutionary path.

b) The European Extremely Large Telescope (E-ELT) is a revolutionary scientific project for a $40 \mathrm{~m}$-class telescope that will allow us to address many of the most pressing unsolved questions about our Universe.

The E-ELT will be the largest optical/near-infrared telescope in the world and will gather 13 times more light than the largest optical telescopes existing today. The E-ELT will be able to correct for the atmospheric distortions (i.e., fully adaptive and diffraction-limited) from the start, providing images 16 times sharper than those from the Hubble Space Telescope. The E-ELT will vastly advance astrophysical knowledge by enabling detailed studies of planets around other stars, the first galaxies in the Universe, super-massive black holes, and the nature of the Universe's dark sector (Gilmozzi \& Spyromilio, 2007).

The final approval of E-ELT occurred at ESO on December 3, 2014 (de Zeeuw, Tamai \& Liske, 2014). On May 25, 2016 ESO Signs Largest Ever Ground-based Astronomy Contract for ELT Dome and Telescope Structure (eso1617 - Organisation Release). An expanded view of the Universe (Science with the European Extremely Large Telescope) can be found in http://www.eso.org/sci/facilities/eelt/docs/.

c) The James Webb Space Telescope (JWST) will be a giant leap forward in our quest to understand the Universe and our origins. JWST will examine every phase of cosmic history: from the first luminous glows after the Big Bang to the formation of galaxies, stars, and planets to the evolution of our own solar system (https://jwst.nasa.gov/science.html).

d) The THESEUS mission is designed to vastly increase the discovery space of the high energy transient phenomena over the entirety of cosmic history (http://www.isdc.unige.ch/theseus/).

The main scientific goals of the proposed mission are to:

i) Explore the Early Universe (cosmic dawn and reionization era) by unveiling a complete census of the Gamma-Ray Burst (GRB) population in the first billion years.

ii) Perform an unprecedented deep monitoring of the $\mathrm{X}$-ray transient Universe.
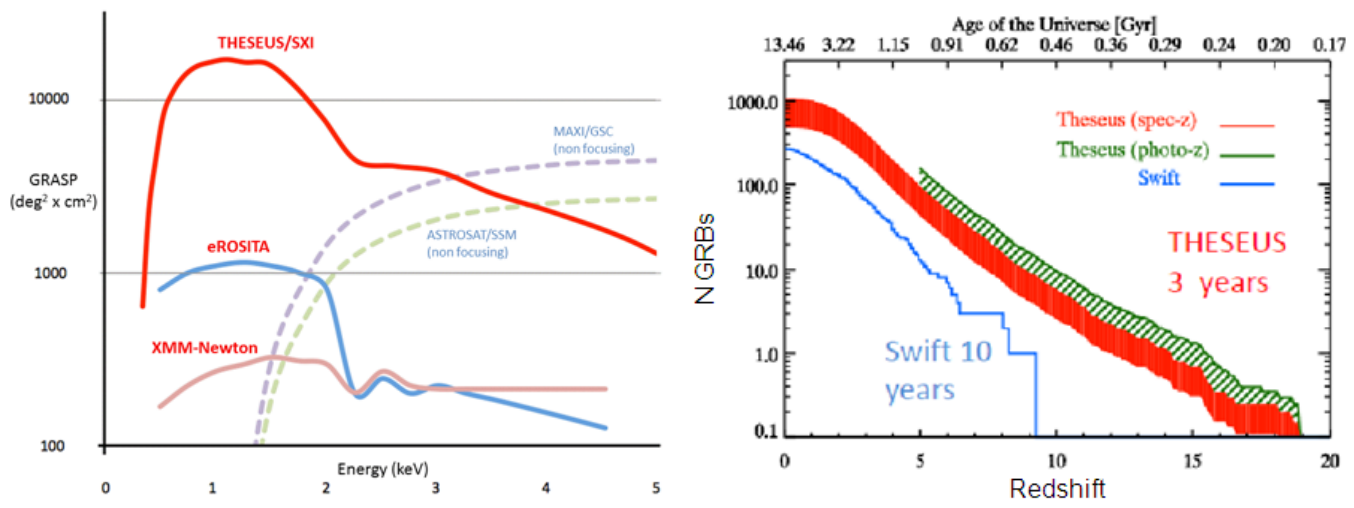

Figure 17: (Left panel: the GRASP (FoV versus Effective Area) as function of energy; right panel: the cumulative distribution of GRBs with redshift determination as a function of the redshift for Swift (in $10 \mathrm{yr}$ ) and the prediction for THESEUS (in $3 \mathrm{yr}$ ) (http://www.isdc.unige.ch/theseus/). 
Figure 17 shows the GRASP FoV versus Effective Area) as function of energy (left panel) and the cumulative distribution of GRBs with redshift determination as a function of the redshift for Swift (in $10 \mathrm{yr}$ ) and the prediction for THESEUS (in $3 \mathrm{yr}$ ) (right panel).
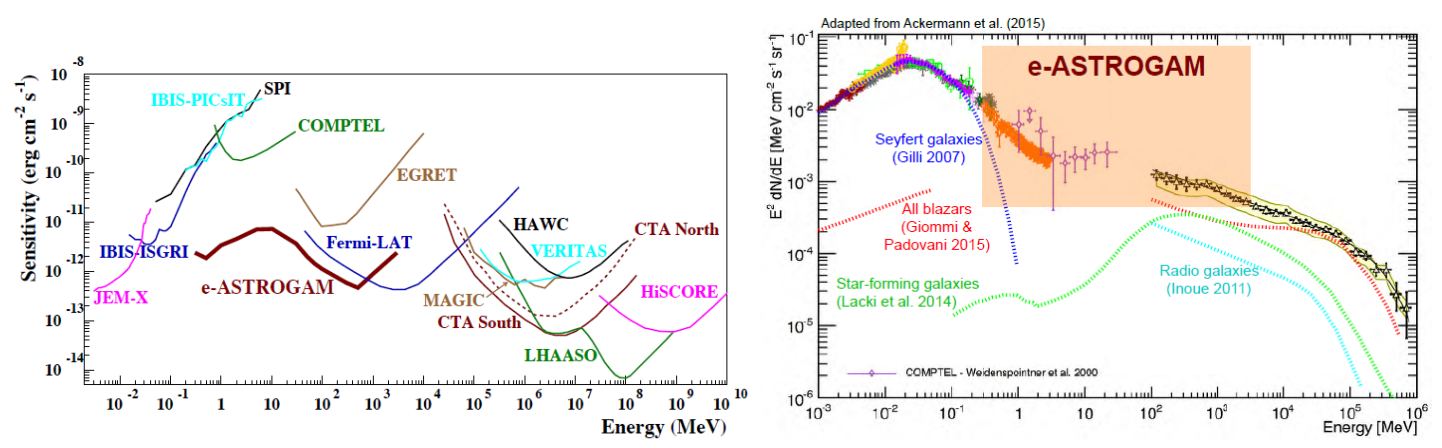

Figure 18: Left panel: the sensitivity of e-ASTROGAM compared with those of the past, present and future experiments; right panel: the compilation of measurements of extragalactic sources between $1 \mathrm{keV}$ and 820 $\mathrm{GeV}$, The semitransparent band indicates the energy region in which e-ASTROGAM will strongly improve on present knowledge (De Angelis, et al., 2017).

e) e-ASTROGAM (enhanced ASTROGAM) is a breakthrough Observatory space mission, with a detector composed by a Silicon tracker, a calorimeter, and an anticoincidence system, dedicated to the study of the non-thermal Universe in the photon energy range from $0.3 \mathrm{MeV}$ to $3 \mathrm{GeV}$ the lower energy limit can be pushed to energies as low as $150 \mathrm{keV}$, albeit with rapidly degrading angular resolution, for the tracker, and to $30 \mathrm{keV}$ for calorimetric detection. The mission is based on an advanced space-proven detector technology, with unprecedented sensitivity, angular and energy resolution, combined with polarimetric capability. Thanks to its performance in the $\mathrm{MeV}-\mathrm{GeV}$ domain, substantially improving its predecessors, e-ASTROGAM will open a new window on the non-thermal Universe, making pioneering observations of the most powerful Galactic and extragalactic sources, elucidating the nature of their relativistic outflows and their effects on the surroundings. With a line sensitivity in the MeV energy range one to two orders of magnitude better than previous generation instruments, e-ASTROGAM will determine the origin of key isotopes fundamental for the understanding of supernova explosion and the chemical evolution of our Galaxy. The mission will provide unique data of significant interest to a broad astronomical community, complementary to powerful observatories such as LIGO-Virgo-GEO600-KAGRA, SKA, ALMA, E-ELT, TMT, LSST, JWST, Athena, CTA, IceCube, KM3NeT, and the promise of eLISA (De Angelis, et al., 2017).

Figure 18 shows: in the left panel the sensitivity of e-ASTROGAM compared with those of the past, present and future experiments; in the right panel the compilation of measurements of extragalactic sources between $1 \mathrm{keV}$ and $820 \mathrm{GeV}$, The semitransparent band indicates the energy region in which e-ASTROGAM will strongly improve on present knowledge (De Angelis, et al., 2017).

f) The GAMMA-400 gamma-ray telescope: the next absolutely necessary step in the development of extraterrestrial high-energy $\gamma$-ray astronomy is the improvement of the physical and technical characteristics of $\gamma$-ray telescopes, especially the angular and energy resolutions. Such a 
new generation telescope will be GAMMA-400, which will be installed onboard the Russian space observatory.

The GAMMA-400 gamma-ray telescope is intended to measure the fluxes of gamma-rays and cosmic-ray electrons and positrons in the energy range from $100 \mathrm{MeV}$ to several TeV. Such measurements concern the following scientific tasks: investigation of point sources of gamma-rays, studies of the energy spectra of Galactic and extragalactic diffuse emission, studies of gamma-ray bursts and gamma-ray emission from the Sun, as well as high precision measurements of spectra of high-energy electrons and positrons. Also the GAMMA- 400 instrument provides the possibility for protons and nuclei measurements up to knee. But the main goal for the GAMMA-400 mission is to perform a sensitive search for signatures of dark matter particles in high-energy gamma-ray emission (Topchiev et al., 2016a,b, and the references therein).

The GAMMA-400 will operate in the highly elliptic orbit continuously for a long time with the unprecedented angular $\left(\sim 0.01^{\circ}\right.$ at $\left.\mathrm{E}_{\gamma}=100 \mathrm{GeV}\right)$ and energy $\left(\sim 1 \%\right.$ at $\left.\mathrm{E}_{\gamma}=100 \mathrm{GeV}\right)$ resolutions better than the Fermi-LAT, as well as ground $\gamma$-ray telescopes, by a factor of 5-10. GAMMA-400 will permit to resolve $\gamma$-rays from annihilation or decay of dark matter particles, identify many discrete sources (many of which are variable), to clarify the structure of extended sources, to specify the data on the diffuse emission (Topchiev et al., 2017).

g) The Large Hadron Collider (LHC) is the world's largest and most powerful particle accelerator (https://home.cern/topics/large-hadron-collider). It first started up on 10 September 2008, and remains the latest addition to CERN's accelerator complex. The LHC consists of a $27 \mathrm{~km}$ ring of superconducting magnets with a number of accelerating structures to boost the energy of the particles along the way. Figure 19 shows a partial view of the tunnel hosting the accelerator.

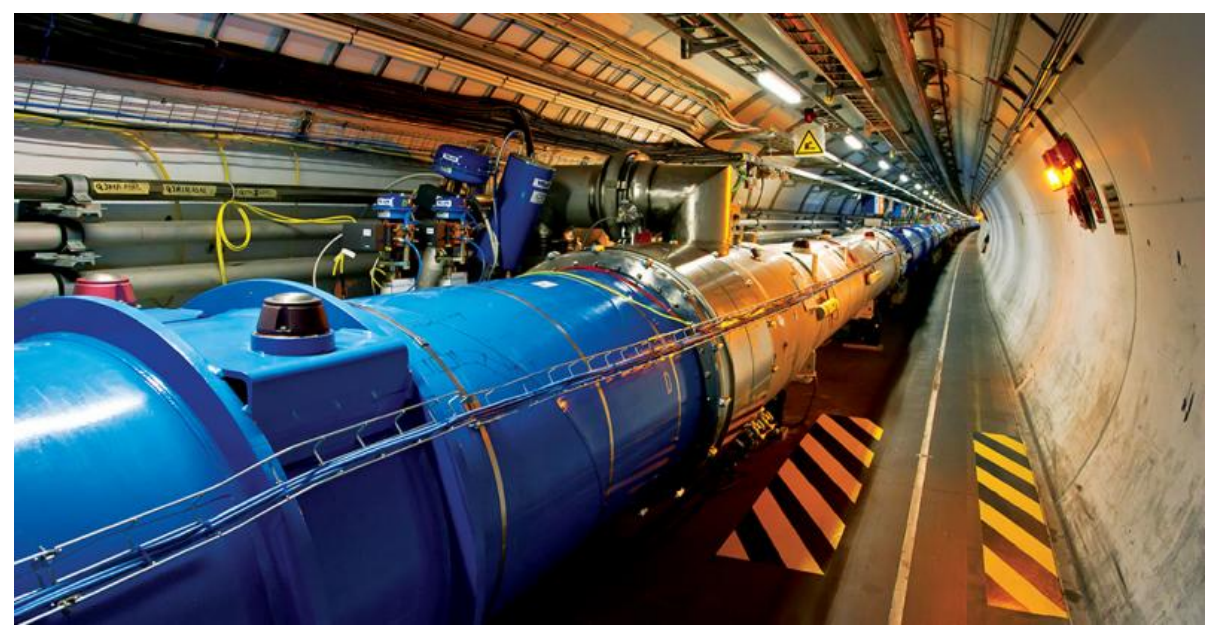

Figure 19: The Large Hadron Collider is the world's largest and most powerful particle accelerator (Image: CERN, at https://home.cern/topics/large-hadron-collider).

The LHC will answer some of the fundamental open questions in physics, concerning the basic laws governing the interactions and forces among the elementary objects, the deep structure of space and time, and in particular the interrelation between quantum mechanics and general relativity. Data is also needed from high-energy particle experiments to suggest which versions of current scientific models are more likely to be correct - in particular to choose between the Standard Model 
and Higgs-less model and to validate their predictions and allow further theoretical development. Many theorists expect new physics beyond the Standard Model to emerge at the TeV energy level, as the Standard Model appears to be unsatisfactory. Issues explored by LHC collisions include i) the mass of elementary particles being generated by the Higgs mechanism; ii) supersymmetry, an extension of the Standard Model and Poincaré symmetry; iii) extra dimensions, as predicted by various models based on string theory; iv) the nature of the dark matter that appears to account for $\sim 27 \%$ of the mass-energy of the universe; v) answer to the question if the electroweak force and the strong nuclear force are just different manifestations of one universal unified force, as predicted by various Grand Unification Theories; vi) why the fourth fundamental force (gravity) is so many orders of magnitude weaker than the other three fundamental forces; vii) are there additional sources of quark flavour mixing, beyond those already present within the Standard Model?; viii) why are there apparent violations of the symmetry between matter and antimatter?; ix) what are the nature and properties of quark-gluon plasma, thought to have existed in the early universe and in certain compact and strange astronomical objects today?

Some important results obtained with the LHC have been discussed by e.g. Giovannelli \& Sabau-Graziati (2018, and the references therein).

h) The BICEP (Background Imaging of Cosmic Extragalactic Polarization) and the Keck Array are a series of cosmic microwave background (CMB) experiments. They aim to measure the polarization of the $\mathrm{CMB}$; in particular, measuring the $\mathrm{B}$-mode of the $\mathrm{CMB}$. The experiments have had four generations of instrumentation, consisting of BICEP1, BICEP2, the Keck Array, and BICEP3. These experiments are observing from the South Pole, and their aims are to discover signatures of Inflation by actually detecting the Cosmic Gravitational Background (CGB) via its weak imprint as the unique $\mathrm{B}$-mode polarization signature of the $\mathrm{CMB}$, directly probing the Universe at an earlier time than ever before. Each generation represents a large increase in sensitivity to Bmode polarization. BICEP1 observed from 2006-2008 with 98 detectors, BICEP2 began observing in the beginning of 2010 with 512 detectors, and the first three of five Keck Array telescopes began observing in the beginning of 2011, each with 512 detectors. The final two Keck Array receivers were deployed during the summer season of 2012. BICEP3, with a total of 2,560 detectors, has been operational since May 2016.

The main goal of BICEP experiments is to test the validity of the theory of the Inflation (Keating et al., 2003; Ogburn IV et al., 2010).

In particular, if Inflation happened immediately after the Big Bang, it would have produced turbulence in the structure of space-time itself-gravitational waves like the kind LIGO detected recently. While these waves would be too weak for LIGO to see, they would twist the orientation of the light, which is known as polarization.

A discussion about the results coming from BICEP2/Keck-Array with comments on their validity has been reported by e.g. Giovannelli \& Sabau-Graziati (2018, and the references therein).

Of course the list of big experiments is far to be complete, but it is enough to show to the reader the efforts that the international scientific community are facing both for determining the frontier scientific tests to validate the current theories and for the difficulties in providing sufficient budgets for their realization. 


\subsection{Small experiments}

Giovannelli \& Sabau-Graziati (2015b) extensively discussed about the tools necessary for an advance of our knowledge of the physics of the Universe. Among them, I cannot omit the extreme importance of small experiments, like those Space-based: small-, mini-, micro-, nano-, and cubesatellites, and those Ground-based: small-telescope, and Robotic-telescopes.

Castro-Tirado (2010a) in his review "Robotic Autonomous Observatories: A Historical Perspective" presented a historical introduction to the field of Robotic Astronomy, discussing the basic definitions, the differing telescope control operating systems, observatory managers, as well as a few current scientific applications in that time.

The number of automatic astronomical facilities worldwide continues to grow, and the level of robotization, autonomy, and networking is increasing as well. This has a strong impact in many astrophysical fields, like the search for extrasolar planets, the monitoring of variable stars in our Galaxy, the study of active galactic nuclei, the detection and monitoring of supernovae, and the immediate followup of high-energy transients such as gamma-ray bursts (Castro-Tirado, 2008, 2010b).

The number of Robotic Autonomous Observatories (RAOs) has rapidly grown. Figure 20 shows the location of more than 100 RAOs worldwide (Castro Cerón, 2011). They are providing excellent results which should be impossible to obtain with the larger telescopes subject to strict scheduling, and in any case not available for long term runs of observations.

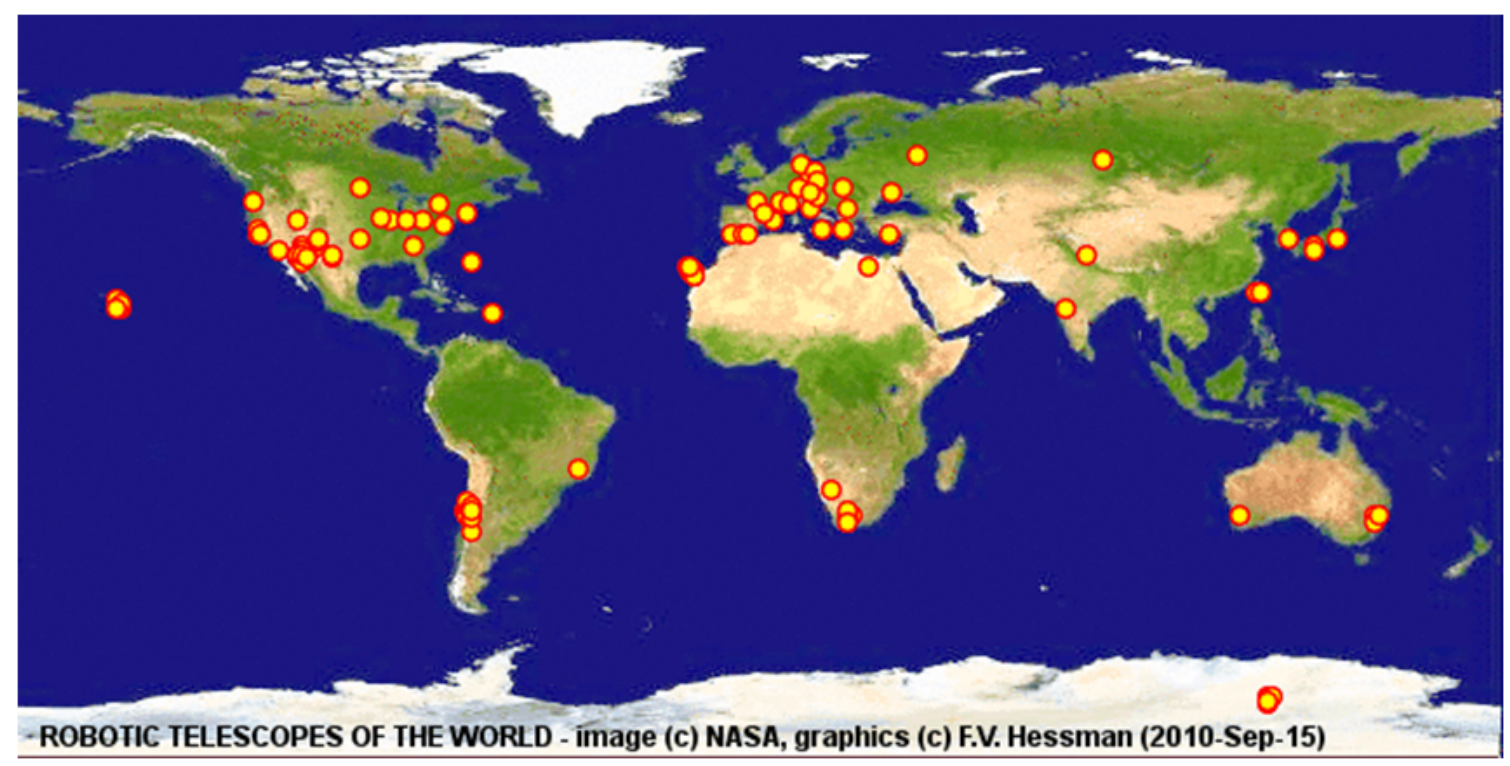

Figure 20: The Robotic Autonomous Observatories worldwide (adopted from Castro Cerón (2011) after Hessman (2001a,b).

The most important news about the many scientific results obtained with the RAOs can be found in the proceedings of the series of Workshops on Robotic Autonomous Observatories (Bloom, Castro-Tirado, Hanlon \& Kotani, 2010; Guziy, Pandey, Tello \& Castro-Tirado, 2012; Tello, Riva, Hiriart \& Castro-Tirado, 2014; Caballero-García, Pandey, Hiriart \& Castro-Tirado, 2016). 
Just for giving to the reader a short panorama about the many small ground- and space-based experiments, not necessarily autonomous, we list the following:

a) MITSuME (Multicolor Imaging Telescope for Survey and Monstrous Explosions) has been built to perform Multi-color photometry of NIR/optical afterglow covering the wavebands from $\mathrm{K}_{s}$ to g' allowing the photometric redshift measurements up to $\mathrm{z} \sim 10$. Two $50 \mathrm{~cm}$ optical telescopes are built at Akeno, Yamanashi in eastern Japan, and at OAO, Okayama in western Japan. Each telescope has a Tricolor Camera, which allows us to take simultaneous images in $\mathrm{g}^{\prime}, \mathrm{R}_{c}$, and $\mathrm{I}_{c}$ bands. These telescopes respond automatically to GCN alerts and start taking series of tricolor images, which are immediately processed through the analysis pipeline on site. The pipeline consists of source finding, catalog matching, sky coordinates mapping to the image pixels, and photometry of the found sources. An automated search for an optical counterpart is performed. While waiting for GRBs, the MITSuME Telescopes automatically patrol pre-selected interesting objects such as AGNs and galactic transients for multiwavelength studies with Fermi (GLAST) and MAXI (Shimokawabe et al., 2009).

b) The CHASE (CHilean Automatic Supernova sEarch) project began in 2007 (Pignata et al., 2009) with the goal to discover young, nearby southern supernovae in order to i) better understand the physics of exploding stars and their progenitors, and ii) refine the methods to derive extragalactic distances. During the first four years of operation, CHASE has produced more than 130 supernovae, being the most successful project of its type in the southern hemisphere (Hamuy et al., 2012).

c) The Russian global network of telescopes robot MASTER (Lipunov et al., 2010). MASTER is very fast positioning alert, follow up and survey twin telecopes Global network with own real-time auto-detection software. MASTER goal is One Sky in One Night up to 20-21 mag. The network is spread along the whole world. In the following are reported the MASTER Net Sites: i) MASTER-Amur: Russia, near Blagoveschensk. Blagoveschensk State Pedagogic University. ii) MASTER-Tunka: Russia, near Irkutsk. Applied Physics Institute, Irkutsk State University. iii) MASTER-Ural: Russia, near Ekaterinburg, Since 2008. Kourovka Astronomical Observatory, Ural State University. iv) MASTER-Kislovodsk: Russia, Near Kislovodsk. Kislovodsk Solar Station of the Pulkovo Observatory, Sternberg Astronomical Institute, Lomonosov Moscow State University. v) MASTER-SAAO: South Africa, Sutherland, since 2014. South African Astonomical Observatory (SAAO). vi) MASTER-IAC: Spain, Canarias Islands, since 2015 The Instituto de Astrofísica de Canarias (IAC). vii) MASTER-OAFA: Argentina, since 2012 Observatorio Astronomico Felix Aguilar (OAFA), Instituto de Ciencias Astronomicas de la Tierra y del Espacio (ICATE), National University of San Juan. viii) MASTER-Progenitor: Russia, Moscow, Alexander Krylov Observatory, Since 2002.

d) Very small satellites for multifreqyency astrophysics have been discussed by Hudec (2017). About the small satellites we can assist to a strong competition (typically for ESA missions, 60 proposals for 1 satellite), and moreover all the system is affected by funding problems.

The development of the Pico (Cube) and Nanosatellites is running at many Universities, mostly with involvement of students for evident goals of education.

The standard size for a CubeSat is 1 Liter Volume, i.e. $10 \times 10 \times 10 \mathrm{~cm}^{3}$ and typically a weight of $\sim 1.3 \mathrm{~kg}$. Multiple modules are possible, i.e. 3 Units $=3$ modules/units, i.e. $10 \times 10 \times 30 \mathrm{~cm}^{3}$, 
typically up to 12 Units.

The range of weight of Picosatellites is 0.1-1 kg, Femtosatellites 10-100 g, Nanosatellites 1-10 $\mathrm{kg}$, Microsatellites 10-100 kg.

Recent technological progress allows their use in any field of astrophysics.

Undoubtedly MASTER contributions to transient alerts in Astronomer's telegrams is fundamental. For instance in the period 2013-2014, MASTER contribution is of order 25\% of the total as shown in Fig. 21 (after Buckley, 2015).

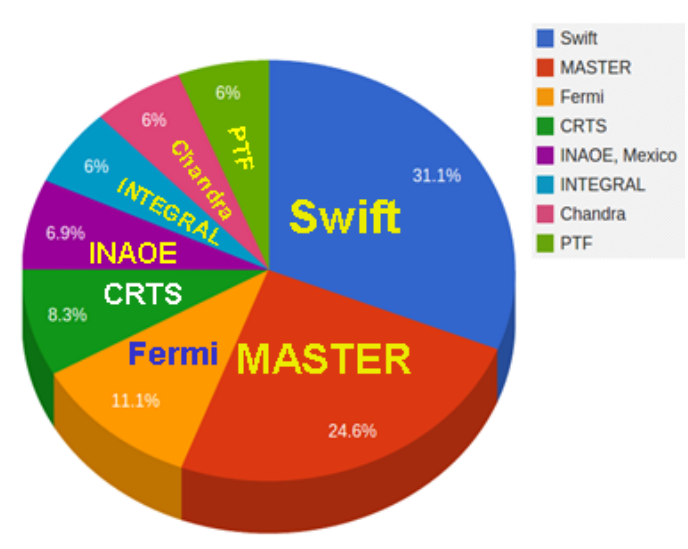

Figure 21: Contribution of different space- and ground-based experiments to the transient alerts in Astronomer's telegrams in the period 2013-2014 (after Buckley, 2015).

Figure 22 shows a suggestive outline of $684 \mathrm{CV}$ discovered by MASTER-NET (adopted from Bukley, 2017b).

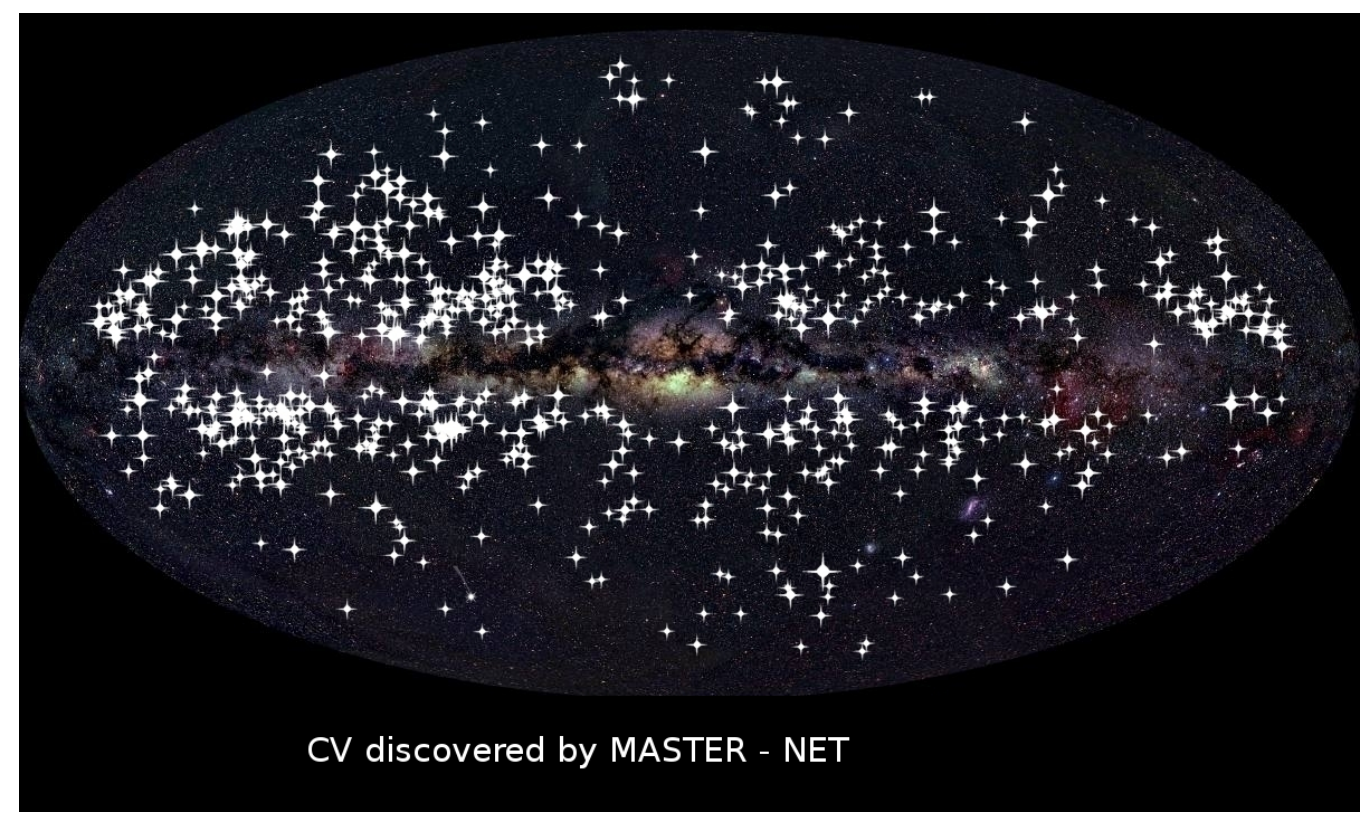

Figure 22: MASTER CV distribution of 684 systems discovered (adopted from Buckley, 2017b). 
Though we have not shown a complete list of small experiments both space- and groundbased, we are able to affirm that small telescopes are unreplaceable tools complementary to larger telescopes and to bigger ground- and space-based multifrequency experiments.

\section{Renewed Interest for Cataclysmic Variables}

Before the advent of ROSAT X-ray satellite, MCVs were relegated to a subsection of conferences about CVs that were mainly concentrated on NMCVs. The ROSAT satellite discovered many MCVs that even menaced to overthrow our understanding of the secular evolution of "normal' $\mathrm{CVs}$ by appearing - apparently inexplicable - in the so-called 'period gap' in the orbital-distribution of CVs (e.g. Vrielmann \& Cropper, 2004). But in spite of this, CVs were not considered, in general, for many years as principal targets of high energy X-ray experiments.

At the beginning of the nineties of the last century, acceleration of particles by the rotating magnetic field of the WD in intermediate polars in the propeller regime - AE Aqr - detected by ground-based Cherenkov telescopes in the TeV passband (e.g. Meintjes et al. 1992), and TeV emission from the polar AM Her detected by ground-based Cherenkov telescopes (Bhat et al. 1991) - measurements never confirmed - were the main reasons of renewed interest for CVs in the high energy astrophysicists community.

Indeed, many measurements were later performed after the news about the possibility of VHE emission from AE Aqr. Chadwick et al. (1995) reported an excess of pulsed VHE $\gamma$ from AE Aqr on 1993 October 11, with the University of Durham VHE $\gamma$-ray telescope at Narrabri, Australia. The burst lasted $\sim 4200 \mathrm{~s}$ at phase $0.62-0.74$ of the $9.88 \mathrm{~h}$ orbital period. Lang et al. (1998) observed AE Aqr for a total of 68.7 hours over the epoch 1991-95 using the Whipple Observatory $10-\mathrm{m} \gamma$-ray telescope. No evidence for any steady, pulsed or episodic $\mathrm{TeV}$ emission. Sidro et al. (2008) reported the observations of AE Aqr with the MAGIC telescope, performed in August 2005 during four consecutive nights within the context of a quasi-simultaneous multi-wavelength campaign covering the radio, optical, UV, and X-ray ranges. The analysis of these data revealed no evidence for any steady or pulsed $\gamma$-ray emission.

Meintjes, Oruru \& Odendaal (2012) discussed the multifrequency properties of AE Aqr within the framework of its secular evolution and a very effective magnetospheric propeller process. The very peculiar broad band emission from radio to possibly $\mathrm{TeV} \gamma$-rays is a manifestation of the propeller driven spin-down power of the white dwarf, which is $\mathrm{L}_{\text {prop }} \sim 10^{34} \mathrm{erg} \mathrm{s}^{-1}$. This may put $\mathrm{AE}$ Aqr in the class of rotation-powered pulsars, and provide an attractive framework to explain the earlier reports of the transient VHE-TeV $\gamma$-ray emission. It is remarkable to notice that AE Aqr lies just in the propeller area in the Lipunov's diagram reported in Fig. 23.

Terada et al. $(2008,2010)$ performed a high sensitive search for possible hard X-ray emission from the fast rotating WD, AE Aquarii with Suzaku. The object has a magnetic field with $10^{5}$ Gauss and a rapid spin period of $33 \mathrm{sec}$. From $100 \mathrm{ksec}$ dataset of Suzaku XIS and HXD, they have discovered spiky pulsations like pulsars in the hard X-ray band of over $4 \mathrm{keV}$, in addition to the well-known thermal modulation in the softer band. In order to account for the hard X-ray signals with the HXD, the X-ray spectrum requires an additional hard X-ray component on the well-known thermal emissions with temperatures of 0.5 and $2.9 \mathrm{keV}$. Combined with results from timing analyses, spectral shapes and flux, they concluded that the hard X-ray pulsations should be 


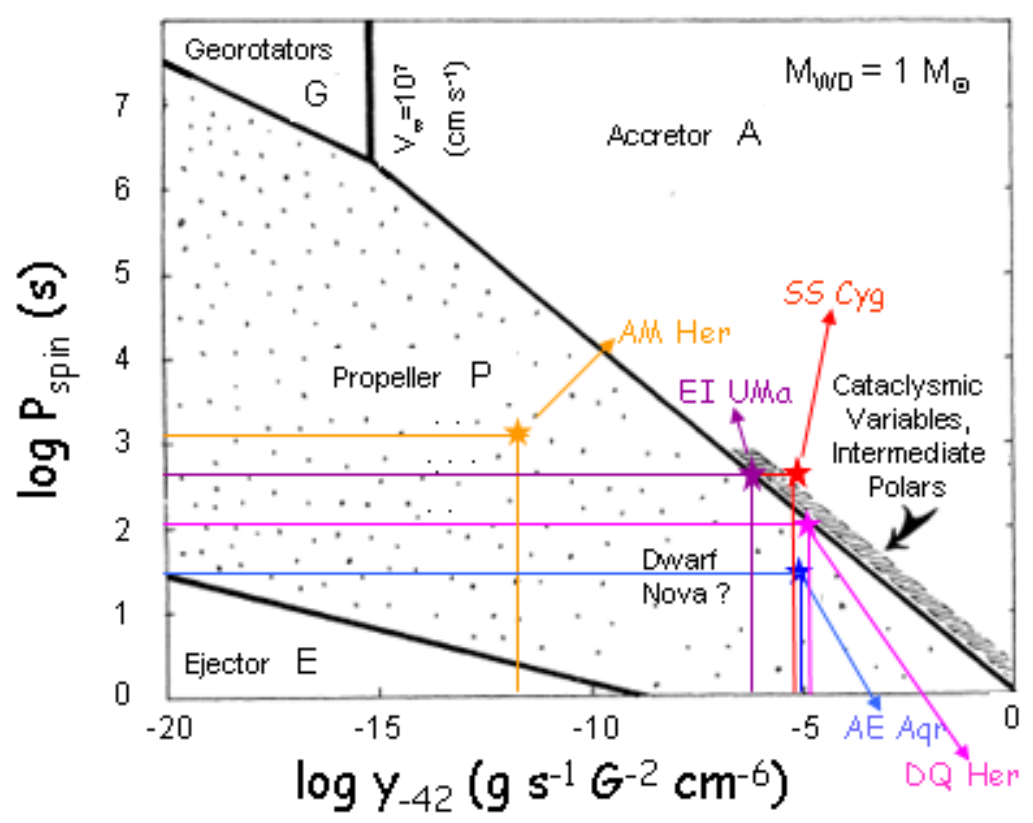

Figure 23: The positions of several CVs in Lipunov's diagram calculated for $1 \mathrm{M}_{\odot}$ white dwarf (adopted from Giovannelli \& Sabau-Graziati, 2015a after Lipunov, 1987).

a non-thermal origin, for example, possible Synchrotron emission with sub MeV electrons. Thus, the object must be a first discovery of a WD equivalent of a neutron star pulsar (e.g. Tsuru, 2009). Tsuru (2008) presented such result in a plot taken by Hillas (1984) shown in Fig. 24.

Aleksic et al. (2014) with the MAGIC experiment searched for steady $\gamma$-ray emission from AE Aqr during $12 \mathrm{~h}$ as part of a multi-wavelength campaign carried out between May and June 2012 covering the optical, X-ray, and $\gamma$-ray ranges. They did not find any significant $\gamma$-ray emission from AE Aqr in any of the searches performed.

The INTEGRAL observatory, until the beginning of 2007, had observed over 70 percent of the sky, with a total exposure time of 40 million seconds. Bird et al. (2007) published the third INTEGRAL catalogue of gamma-ray sources. It contains a total of 421 gamma-ray objects. Most have been identified as either binary stars in our Galaxy containing exotic objects such as black holes and neutron stars, or active galaxies, far away in space. But a puzzling quarter of sources remain unidentified so far. They could be either star systems enshrouded in dust and gas, or CVs. INTEGRAL observes in the gamma-ray band so it can see through the intervening material. It has demonstrated that it can discover sources obscured at other wavelengths. One surprise has been the efficiency with which INTEGRAL has detected just one minor subclass CVs, the so-called IPCVs. Initially astronomers were not sure that CVs would emit gamma rays. Indeed, INTEGRAL has already shown that only about one percent of them do. This fact overbearingly renewed the interest for CVs, apparently fallen into disgrace in favour of binary systems containing either neutron stars or black holes. The fourth IBIS/ISGRI catalog reports 331 additional sources when compared to the third catalog. Of these, 120 are associated with extragalactic sources, while only 25 are associated with known Galactic sources, and the remainder are so far unidentified (Bird et al. 2010). CVs constitute $\sim 5 \%$ of the total sources. 


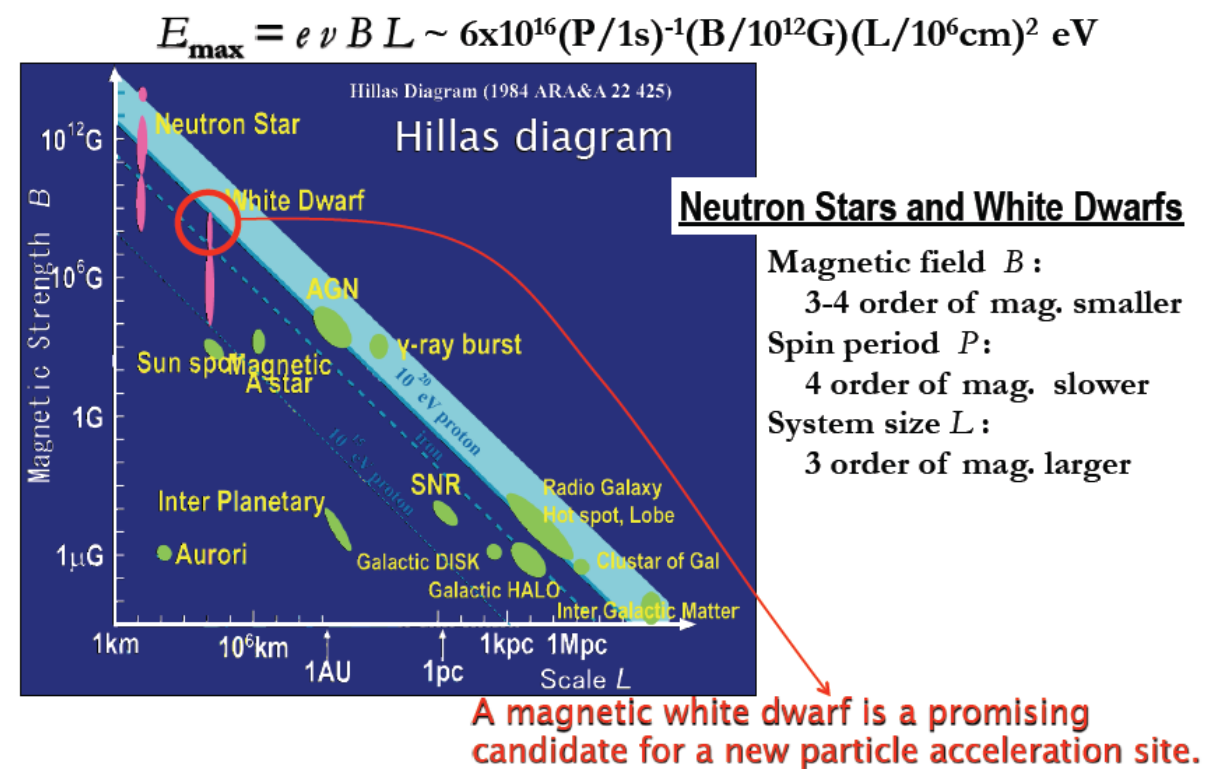

Figure 24: AE Aqr: a possible candidate for a new particle acceleration site (adopted from Tsuru, 2008, after Hillas, 1984).

Moreover, since the CVs measured by the INTEGRAL observatory are magnetic in nature, the interest for such class of objects has been addressed to evolutionary problems.

The long-standing fundamental predictions of evolution theory are finally being tested observationally. All facets of the accretion process in CVs, including variability, disk winds and jets, are universal with accreting WDs, neutron stars, and black holes (Knigge, 2010, 2011). Knigge, Baraffe \& Patterson (2011) extensively discussed the reconstruction of the complete evolutionary path followed by CVs, based on the observed mass-radius relationship of their donor stars, following Knigge (2006) that discussed the observational and theoretical constraints on the global properties of secondary stars in CVs using the semi-empirical CV donor sequence, and concluded that most CVs follow a unique evolutionary track.

In the standard model of CV evolution, angular-momentum-loss (AML) below the period gap are assumed to be driven solely by gravitational radiation (GR), while AMLs above the gap are usually described by a magnetic bracking (MB) (Rappaport, Verbunt \& Joss (1983). Knigge, Baraffe \& Patterson (2011) with their revised model, found the optimal scale factors $\mathrm{f}_{\mathrm{GR}}=2.47$ below the gap and $\mathrm{f}_{\mathrm{MB}}=0.66$ above, whilst the standard model gives $\mathrm{f}_{\mathrm{GR}}=\mathrm{f}_{\mathrm{MB}}=1$. This revised model describes the mass-radius data much better than the standard model.

The sub-class of $\mathrm{CV}$ s, named Classical Novae $(\mathrm{CNe})$, which are the third more powerful stellar explosions in a galaxy, have been observed as close as a kpc and as far as galaxies in Fornax cluster. The time to report on the recent renaissance in studies on $\mathrm{CNe}$ thanks to observations with $8-10 \mathrm{~m}$ class telescopes, high resolution spectroscopy, in synergy with observations from space carried out with Swift, XMM, Chandra, HST, and Spitzer, coupled with recent advances in the theory of the outburst, seems now in order. Moreover, the possible connection among some CV-types and SNe-Ia will definitively justify the renewed interest about CVs. 


\subsection{Dwarf novae as pulsars}

As announced in the abstract it is important to mention the big news about the discovery of polarization in AR Sco.

AR Sco is a $3.56 \mathrm{~h}$ binary system consisting of a rapidly spinning $\left(\mathrm{P}_{\text {spin }}=117 \mathrm{~s}\right)$ white dwarf and an M-type main-sequence companion star. It was discovered to pulse across the electromagnetic system, from UV to radio, dominantly at the $\sim 118 \mathrm{~s}$ beat period (Marsh et al., 2016).

The optical pulsed emission was discovered to be highly linearly polarized, up to $40 \%$ (Buckley et al., 2017), which led to the interpretation that AR Sco is the first white dwarf pulsar.

Indeed, the pulsed luminosity of AR Sco is powered by the spin-down of the rapidly-rotating white dwarf which is highly magnetized (up to $\sim 500 \mathrm{MG}$ ). The morphology of the modulated linear polarization is similar to that seen in the Crab pulsar, albeit with a more complex waveform owing to the presence of two periodic signals of similar frequency. Magnetic interactions between the two component stars, coupled with synchrotron radiation from the white dwarf, power the observed polarized and non-polarized emission. AR Sco is therefore the first example of a white dwarf pulsar (Buckley et al., 2017).

Various models consisting of magnetic interactions between the two stars, accelerating relativistic electrons producing synchrotron radiation and magnetohydrodynamic interactions have been put forward to explain the power behind the observed polarized and non-polarized emission, e.g. Marsh et al. (2016), Buckley et al. (2017), Takata, Yang \& Cheng (2017), Geng, Zhang \& Huang (2016), and Katz (2017).

The discovery of a first white dwarf showing typical characteristics of pulsars, encourages the community of high energy physicists to undertake precise measurements to reveal other systems similar to AR Sco, which for reasons of evolutionary logic must exist.

\section{Classical and Recurrent Novae}

Classical novae are expected to recur on timescales from 100,000 years to just a few decades. The most important physical parameters controlling this recurrence timescale are the WD mass, and the mass accretion rate from the secondary (e.g. Yaron et al. 2005). Once classical nova (CN) is recorded more than once, it can be designated as "recurrent" (RN). Since the WD and the binary system remain intact after an outburst, it is possible that classical novae may actually be the same as recurrent novae if observed over a long enough time period. While the interval between outbursts of recurrent novae range from 10 to 100 years, it has been estimated that the time interval for classical novae would range from about 30,000 years for a $1.3 \mathrm{M}_{\odot}$ WD to 100,000 years for a $0.6 \mathrm{M}_{\odot} \mathrm{WD}$. Given long enough - it is expected that all classical novae will be observed as recurrent novae.

The long term behaviour of classical old novae, and the optical behaviour of $\mathrm{CNe}$ in outburst were discussed by Bianchini (1990), and Seitter (1990), respectively. The books by Cassatella \& Viotti (1990) and by Bode \& Evans (2008) are very useful for studying the physics of classical novae.

Recurrent novae are a rare sub-class of CVs; WDs accreting material from a binary companion in which more than one classical nova-type outburst has been observed (see the book of Hellier, 2001 for a comprehensive review of CVs). Nova outbursts are suspected to be due to a thermonu- 
clear runaway on the surface of the WD, which releases huge amounts of thermal energy once a critical pressure is reached at the base of the shell of accreted material.

One of the most interesting RNe is RS Ophiuchi (RS Oph). It is an amazingly prolific recurrent nova, with recorded outbursts in 1898, 1907, 1933, 1945, 1958, 1967, 1985 and 2006 (Schaefer 2010). The short time between outbursts ( $\sim 20$ yrs) suggests that RS Oph hosts a massive WD accreting material at a significantly high rate.

In the latter paper Schaefer discussed not only RS Oph, but also the photometric histories of all known galactic RNe.

Classical and recurrent nova outbursts have been discussed by Bode (2011a,b) and Evans (2011). The proceedings of a conference about RS Oph and recurrent phenomenon can be very useful for details (Evans et al., 2008). General properties of quiescent novae have been discussed by Warner (2002). The very useful book of Bode \& Evans (2008) about classical novae examines thermonuclear processes, the evolution of nova systems, nova atmospheres and winds, the evolution of dust and molecules in novae, nova remnants, and observations of novae in other galaxies. It includes observations across the electromagnetic spectrum, from radio to gamma rays, and discusses some of the most important outstanding problems in classical nova research.

Of the $\sim 400$ known Galactic classical novae, only 10 of them are recurrent. Eight of them harbour evolved secondary stars, contrary to classical novae that contain main sequence stars (Darnley et al., 2011). They propose a new nova classification based on the evolutionary state of the secondary star, contrary the current schemes based on the properties of outbursts. Such classification contains three groups of novae: i) Main Sequence Nova (MS-Nova); ii) Sub-Giant Nova (SGNova); and iii) Red Giant branch Nova (RG-Nova).

An important not yet resolved problem is connected with the evolution and fate of Classical Novae. Patterson (2014) discussed this crucial problem. Classical novae rise from obscurity to shine among the brightest stars in the Galaxy. The story of how they return to quiescence is still only dimly known. Vast amounts of energy are loosed upon the WD and its companion, and the light curves of post-novae suggest that they take not a few years, but a few thousand years, to return to quiescence. In the meantime, the secondary may experience a lot of heating from the WD's radiation - enough to overwhelm its intrinsic nuclear luminosity. For this purpose he mentioned the case of BK Lyncis - the oldest old nova and a bell-wether for CVs evolution (Patterson et al., 2013). They discussed stellar physics behind this suggestion and proposed how it might be tested by time-series photometry in the months and years (and if possible, centuries) after outburst.

RNe play an important role in the studies of SN Ia progenitors (Surina, Bode \& Darnley, 2011). $\mathrm{RNe}$ are likely progenitors of Type-Ia supernovae.

On the contrary, Shafter et al. (2015) estimated that $\sim 4 \%$ of the nova eruptions seen in M31 over the past century are associated with RNe. A Monte Carlo analysis shows that the discovery efficiency for RNe may be as low as $10 \%$ that for novae in general, suggesting that as many as one in three nova eruptions observed in M31 arise from progenitor systems having recurrence times $\lesssim 100$ yr. For plausible system parameters, it appears unlikely that RNe can provide a significant channel for the production of Type-Ia supernovae.

Important works have been developed about extragalactic nova populations (Shafter et al., 2014). Nova rates have been measured for more than a dozen galaxies spanning a wide range of 
Hubble types. They found that the recurrent nova population in the LMC appears to be higher than that seen in M31 and the Galaxy.

In order to brave this important problem the use of archival data is the only way to answer the big question. Now, huge and comprehensive set of archival RN data go back to 1890 .

Excellent work about the archival data has been promoted by René Hudec, who scanned thousands plates belong to numerous astronomical observatories spread in the whole world (e.g. Hudec, R. \& Hudec, L., 2013). Indeed, the astronomical plate archives represent the only method how to study the behavior of the CVs (and other astrophysical objects in general) over very long (100 years or even more) time intervals, and the only method to go back in time. In addition, huge monitoring times (up to 30,000 hrs of continuous monitoring) are available allowing to detect and to study rare events such as outbursts. The databases allow to study prominent spectra and/or spectral changes as well (Hudec, R. Hudec, L. \& Klíma, M., 2012).

A recent review about galactic and extragalactic novae has been published by Poggiani (2017a), in which she discusses the multifrequency observations that are contributing to understanding the process of explosions and of the long term evolution. She discusses the observations of novae over the electromagnetic spectrum, focusing on the morphology of the decline light curves, the spectroscopic investigations, the long term evolution, the recurrent novae, the gamma ray emission in novae, extragalactic novae, and the gravitational emission of novae.

\section{Progenitors of SN Ia}

It is well accepted by the community that Type-Ia SNe are the result of the explosion of a carbon-oxygen WD that grows to near Chandrasekhar's limit in a close binary system (Hoyle \& Fowler, 1960). But the debate is focussed around the different kinds of progenitors. Indeed, in the past, two families of progenitor models have been proposed. They differ in the mode of WD mass increase. The first family is the so-called single degenerate (SD) model (Whelan \& Iben, 1973), in which the WD accretes and burns hydrogen-rich material from the companion. The second family is the so-called double degenerate (DD) model, in which the merging of two WDs in a close binary triggers the explosion (Webbing, 1984; Iben \& Tutukov, 1984). The two scenarios produce different delay times for the birth of the binary system to explosion. Thus it is hopefully possible to discover the progenitors of Type-Ia SNe by studying their delay time distribution (DDT). The DDT can be determined empirically from the lag between the cosmic star formation rate and Type-Ia SN birthrate.

The energy released through runaway thermonuclear process ejects the majority of the unburnt hydrogen from the surface of the star in a shell of material moving at speeds of up to $1.5 \times 10^{3} \mathrm{~km}$ $\mathrm{s}^{-1}$. This produces a bright but short-lived burst of light - the nova.

Although Type-Ia supernovae appear to have similar origin to classical novae, there are key differences. The most important is that in a classical nova, the thermonuclear runaway occurs only on the surface of the star, allowing the WD and the binary system to remain intact (e.g. Townsley $\&$ Bildsten, 2005). In a Type-Ia supernova, the thermonuclear runaway occurs within WD itself, completely disrupting the progenitor. This is reflected in the amount of energy released in the explosions, with classical novae releasing $\sim 10^{44} \mathrm{erg}$, and Type-Ia supernovae $\sim 10^{51} \mathrm{erg}$. 
The possible progenitors of SN Ia are: i) Recurrent Novae; ii) Symbiotic stars; iii) Super-soft sources; iv) Double WD Binaries; and v) WDs accreting material from red-giant companions.

i) Recurrent Novae are just a subset of ordinary novae that happen to go off more than once per century.

As such, they are binary systems with matter flowing off a companion star onto a WD, accumulating on its surface until the pressure gets high enough to trigger a thermonuclear runaway that is the nova.

Only 10 RNe are known in our Milky Way galaxy, including: U Sco $(1863,1907,1917,1936$, 1945, 1969, 1979, 1987, 1999); T Pyx (1890, 1902, 1920, 1944, 1967); T CrB (1866, 1946); RS Oph $(1898,1907,1933,1945,1958,1967,1985,2006)$.

To recur with $\tau_{\text {rec }}<100$ years, RNe must have: high WD mass $\left(1.2 \mathrm{M}_{\odot}<\mathrm{M}_{\mathrm{WD}}<\mathrm{M}_{\text {Chandra }}\right)$, and high accretion rate $\left(\dot{\mathrm{M}} \sim 10^{-7} \mathrm{M}_{\odot} \mathrm{yr}^{-1}\right)$. SN Ia occurs if: i) the mass ejected for each eruption is less than the mass accreted onto the WD $\left(\mathrm{M}_{\text {ejected }}<\dot{\mathrm{M}} \tau_{\text {rec }}\right)$; ii) the rate of death RNe must be enough to produce the $S N$ Ia rate $\left(R_{R N d e a t h}=R_{S N I a}\right)$, being $R_{R N d e a t h}=N_{R N} \times\left(0.2 M_{\odot} \dot{M}\right)$.

In order to solve the problems we need to know $\tau_{\text {rec }}$ (recurrence time scale) from archive plates, $\mathrm{N}_{\mathrm{RN}}$ (number of RNe in the Milky Way) from archive plates and AAVSO, $\dot{\mathrm{M}}$ (mass accretion rate onto WD) from the average in the last century, $\mathrm{M}_{\mathrm{ejected}}$ (mass ejected in eruption) from pre-eruption eclipse timing.

Some results have been obtained for becoming optimists in solving the problem of SN Ia production. Indeed Schaefer (2011) obtained for CI Aql and U Sco $\mathrm{M}_{\text {ejected }}<<\dot{\mathrm{M}} \tau_{\text {rec }}$ ).

Thus, WDs are gaining mass and the latter RNe will collapse as SN Ia. Moreover, for the Milky Way, M31, and LMC $\mathrm{R}_{\mathrm{RNdeath}} \sim \mathrm{N}_{\mathrm{RN}}$. Then there are enough RNe to supply the Type-Ia $\mathrm{SN}$ events.

ii) Symbiotic Stars contain WDs efficiently accreting material from the secondary star. In most cases they steadily burn H-rich material allowing them to grow in mass. Some of these systems can produce high mass WDs. In symbiotic RNe (SyRNe) the WD mass is already very close to Chandrasekhar's limit. For instance in V 407 Cyg a very massive WD is accreting material at a rate of $\sim 10^{-7} \mathrm{M}_{\odot} \mathrm{yr}^{-1}$ from a Mira-type companion (Mikołajewska, 2011).

iii) Super-soft Sources are probably WDs that accrete material and burn hydrogen. Voss \& Nelemans (2008) discovered an object at the position of the Type-Ia SN2007on in the elliptical galaxy NGC1404 on pre-supernova archival X-ray images. This result favours the accretion model (SD) for this supernova, although the host galaxy is older than the age at which the explosions are predicted in SD models. However, the DD model cannot be ruled out by this event because a hot accretion disc is probably the intermediate configuration of the system, between first WD-WD Roche-lobe contact and explosion (Yoon, Podsiadlowski \& Rosswog, 2007).

Greggio, Renzini \& Daddi (2008) starting from the fact that Type-Ia SN events occur over an extended period of time, following a distribution of delay times (DDT), discussed theoretical DDT functions that accommodate both "prompt" and 'tardy' $\mathrm{SN}$ events derived by empirically-based DDT functions. Moreover such theoretical DDT functions can account for all available observational constraints. The result is that SD/DD mix of SNIa's is predicted to vary in a systematic fashion as function of cosmic time (redshift).

iv) Double WDs Binaries are systems containing two WDs that can merge and giving rise to SN explosion. Yoon, Podsiadlowski \& Rosswog (2007) explored the evolution of the merger 
of two carbon-oxygen (CO) WDs. Their results imply that at least some products of double CO WDs merger may be considered good candidates for the progenitors of Type-Ia SNe. Brown et al. (2011) and Kilic et al. (2011) studied a complete colour-selected sample of double-degenerate binary systems containing extremely low mass (ELM) $\left(\leq 0.25 \mathrm{M}_{\odot}\right)$ WDs. Milky Way disc ELM WDs have a merger rate of $\approx 4 \times 10^{-5} \mathrm{yr}^{-1}$ due to gravitational wave radiation. The ELM WD systems that undergo stable mass transfer can account for about $3 \%$ of AM CVn stars. The most important fact is that the ELM WD systems that may detonate merge at a rate comparable to the estimate rate of underluminous $\mathrm{SNe}$. These $\mathrm{SNe}$ are rare explosions estimated to produce only $\sim 0.2 \mathrm{M}_{\odot}$ worth of ejecta. At least $25 \%$ of ELM WD sample belong to the old tick disc and halo components of our Galaxy. Thus, if merging ELM WD systems are the progenitors of underluminous $\mathrm{SNe}$, transient surveys must find them in both elliptical and spiral galaxies.

v) WDs accreting material from red-giant companions. Observations carried out by Patat et al. (2008) with VLT-UVES allowed to detect circumstellar material in a normal Type-Ia SN. The expansion velocities, densities and dimensions of the circumstellar envelope indicate that this material was ejected from the system prior to the explosion. The relatively low expansion velocities favour a progenitor system where a WD accretes material from a companion star, which is in the red-giant phase at the time of explosion.

Bianco et al. (2011) searched for a signature of a non-degenerate companion in three years of Supernova Legacy Survey data. They found that a contribution from WD/red-giant binary system to Type-Ia SN explosions greater than $10 \%$ at $2 \sigma$, and than $20 \%$ at $3 \sigma$ level is ruled out.

Type-Ia SNe are used as primary distance indicators in cosmology (e.g. Phillips, 2005). Phillips (2011) reviewed the near-infrared (NIR) of Type-Ia SNe concluding that such SNe are essentially perfect standard candles in the NIR, displaying only a slight dependence of peak luminosity on decline rate and colour. Lira (1995) first noted that B-V evolution during the period from 30 to 90 days after V maximum is remarkably similar for all SN Ia events, regardless of light-curve shape. This fact was used by Phillips et al. (1999) to calibrate the dependence of the $B_{\max }-V_{\max }$ and $\mathrm{V}_{\max }-\mathrm{I}_{\max }$ colours on the light curve parameter $\Delta \mathrm{m}_{15}$ (B) which can, in turn, be used to separately evaluate the host galaxy extinction. Using these methods for eliminating the effect of the reddening, they reanalyzed the functional form of the decline rate versus luminosity relationship and gave a value of the Hubble constant of $\mathrm{H}_{0}=63.3 \pm 2.2 \pm 3.3 \mathrm{~km} \mathrm{~s}^{-1} \mathrm{Mpc}^{-1}$.

The use of Type-Ia SNe is also fundamental for determining some cosmological constraints, such as $\Omega_{\mathrm{M}}$ and $\Omega_{\Lambda}$ that fit a $\Lambda \mathrm{CDM}$ models with values of $0.211 \pm 0.034$ (stat) \pm 0.069 (sys) using a set of 252 high-redshift SNe (Guy et al., 2010) and $0.713_{-0.029}^{+0.027}$ (stat) ${ }_{-0.039}^{+0.036}$ (sys) using a set of low-redshift nearby-Hubble-flow SNe (Kowalski et al., 2008), respectively.

In order to explore the difficult topic of the expansion of the Universe it is necessary to know the evolution of metallicity in old Universe that changes the Hubble Diagram shape. The proposed space observatory Super Nova Acceleration Probe (SNAP) is designed to measure the expansion of the Universe and to determine the nature of the mysterious Dark Energy that is accelerating this expansion (Aldering, 2005). SNAP is being proposed as part of the Joint Dark Energy Mission (JDEM) (Stril, Cahn \& Linder, 2010), which is a cooperative venture between NASA and the U.S. Department of Energy. If selected it will be launched before 2020. SNAP cannot achieve its main goal without progenitor/evolution solution. 
The research about the progenotors of SN Ia is of course one of the most important problems, since it is strictly connected with the evolution of CVs. For instance, Maguire et al. (2012) present an analysis of the maximum light, near-ultraviolet (NUV; $2900<\lambda<5500$ ) spectra of 32 lowredshift $(0.001<\mathrm{z}<0.08)$ SNe Ia, obtained with the Hubble Space Telescope (HST) using the Space Telescope Imaging Spectrograph. They combine this spectroscopic sample with high-quality gri light curves obtained with robotic telescopes to measure SN Ia photometric parameters, such as stretch (light-curve width), optical colour and brightness. They confirm and strengthen earlier conclusions regarding the complex behaviour of SNe Ia in the NUV spectral region, but suggest that the correlations found are more useful in putting tighter constraints on the progenitor systems of SNe Ia and how their progenitor channels may vary with host galaxy properties (e.g. metallicity: Kistler et al., 2013) rather than improving the use of SNe Ia as cosmological probes.

Darnley et al. (2014) discussed on the galactic nova progenitor population. They presented a selection of the work and rationale that led to the proposal of a new nova classification scheme based not on the outburst properties but on the nature of the quiescent system. They also outlined the results of a photometric survey of a sample of quiescent Galactic novae, showing that the evolutionary state of the secondary can be easily determined and leading to a number of predictions, including their relevance to extragalactic work and the proposed link to type-Ia SNe.

In order to solve the problem of determining the SN Ia progenitors, it is also important to look at the RNe that show many similarities to $\mathrm{CNe}$, but have had more than one recorded outburst. RNe play an important role as one of the suspected progenitor systems of Type-Ia SNe, which are used as primary distance indicators in cosmology. Thus, it is important to investigate the nature of their central binary systems to determine the relation between the parameters of the central system and the outburst type, and finally ascertain the population of novae that might be available to give rise to the progenitors of Type-Ia SNe. Surina, Bode \& Darnley (2015) adopted a low outburst amplitude as a criterion that may help distinguish $\mathrm{RNe}$ from $\mathrm{CNe}$ and was therefore used to select targets for observations from ground-based observatories including the Liverpool Telescope and the Southern African Large Telescope as well as the full-sky space-based archive of the Solar Mass Ejection Imager (SMEI). They found that at least four objects currently classified as $\mathrm{CNe}$ are possibly RNe candidates based on their quiescent spectra. They also searched the SMEI archive for additional outbursts of bright $\mathrm{CNe}$ that might otherwise have been missed but did not find a conclusive example.

Another possible channel for triggering the explosion of SN Ia is that discussed by Chiosi et al. (2015). They explore the possibility that isolated CO-WDs with mass smaller than the Chandrasekhar limit may undergo nuclear runaway and SN explosion. If this channel could be confirmed it should be possible (i) to explain the star formation rate dependence of the SN Ia rate (e.g. Mannucci, Della Valle \& Panagia, 2006); (ii) to provide some clues to interpreting the observational data on the ejected mass distribution of type-Ia SNe showing a significant rate of non-Chandrasekhar-mass progenitors of mass as low as $0.8 \mathrm{M}_{\odot}$ (Scalzo, Ruiter \& Sim, 2014); and (iii) to account for the SNe exploding inside Planetary Nebulae in alternative to the core-degenerate scenario in which a WD merges with the hot core of an AGB star on a time interval $\leq 10^{8} \mathrm{yr}$ since the WD formation (see Tsebrenko \& Soker, 2015, for more details). With the models of Chiosi et al. (2015), a single CO-WD may reach the explosion stage soon after the formation if sufficiently massive $\left(>1.0 \mathrm{M}_{\odot}\right)$ and sufficiently rich in residual hydrogen $\left(\mathrm{X}_{\mathrm{H}} \simeq 10^{-19}-10^{-20}\right)$. The expected 
time delay after formation can be as low as about a few ten of thousand years.

Williams et al. $(2014,2016)$ report the results of a survey of M31 novae in quiescence. The derived catalog contains data for 38 spectroscopically confirmed novae from 2006 to 2012. They used Liverpool Telescope images of each nova during eruption to define an accurate position for each system. These positions were then matched to archival Hubble Space Telescope (HST) images and they performed photometry on any resolved objects that were coincident with the eruption positions. This in order to facilitate a search for their progenitor systems within archival Hubble Space Telescope (HST) data, with the aim of detecting systems with red giant secondaries (RG-novae) or luminous accretion disks. They found an elevated proportion of nova systems with evolved secondaries that may imply the presence of a much larger population of recurrent novae than previously thought. This would have considerable impact, particularly with regards to their potential as TypeIa SN progenitors. Their results also imply that RG-novae in M31 are more likely to be associated with the M31 disk population than the bulge, indeed the results are consistent with all RG-novae residing in the disk. If this result is confirmed in other galaxies, it suggests any Type-Ia SNe that originate from RG-nova systems are more likely to be associated with younger populations, and may be rare in old stellar populations, such as early-type galaxies.

An important paper by Churazov et al. (2014) reports the first ever detection of ${ }^{56} \mathrm{Co}$ lines at 847 and $1237 \mathrm{keV}$ and a continuum in the $200-400 \mathrm{keV}$ band from the Type-Ia SN2014J in M82 with INTEGRAL observatory. The data were taken between 50th and 100th day since the SN2014J outburst. The line fluxes suggest that $0.62 \pm 0.13 \mathrm{M}_{\odot}$ of radioactive ${ }^{56} \mathrm{Ni}$ were synthesized during the explosion. Line broadening gives a characteristic ejecta expansion velocity $\mathrm{V}_{\mathrm{e}} \sim 2100 \pm 500$ $\mathrm{km} \mathrm{s}^{-1}$. The flux at lower energies (200-400 keV) is consistent with the three-photon positronium annihilation, Compton downscattering and absorption in the $\sim 1.4 \mathrm{M}_{\odot}$ ejecta composed from equal fractions of iron-group and intermediate-mass elements and a kinetic energy $\mathrm{E}_{\mathrm{k}} \sim 1.4 \times 10^{51}$ erg. All these parameters are in broad agreement with a "canonical" model of an explosion of a Chandrasekhar-mass WD, providing an unambiguous proof of the nature of Type-Ia SNe as a thermonuclear explosion of a solar mass compact object. Late optical spectra (day 136 after the explosion) show rather symmetric Co and Fe line profiles, suggesting that, unless the viewing angle is special, the distribution of radioactive elements is symmetric in the ejecta (Churazov et al., 2015).

For comments and prospects about Type-Ia SN science in the decade 2010-2020 see the paper by Howell et al. (2009).

\section{Some Open Questions}

Several fundamental questions concerning CVs still remain waiting for a proper answer. Here we will present briefly only some of them.

One of them is the lack of a coherent classification, especially for NLs. On the other hand, in gross features and in most respects, DN and NLs, as well as quiescent novae, are almost indistinguishable, although, in addition to their different outbursts' behaviour, there appear to be some further minor differences which are not yet understood (see Hack \& la Dous 1993). The question arises of whether the outburst behaviour, the current basis of almost all classification is really a suitable criterion for sorting CVs in physically related groups. There are also too many exceptions, 
either systems that do not fit in any particular group or that can be included in several of them, to be able to render the observational behaviour, at least as it is used at the present, suitable.

Could CVs be considered simply gravimagnetic rotators? This should be the most suitable approach for studying them from a physical point of view.

Studies of rotational equilibria of MCVs predict that IPCVs will evolve either into PCVs or into low field strength polars - presumably unobservable, and possibly EUV emitters - depending on their magnetic moments and orbital periods. Indeed, there are systems, like EX Hya-type, having magnetic moment similar to IPCVs above the 'period gap' and comparable to the weakest field AM Her-like systems.

Moreover, the detection of several SW Sex systems having orbital periods inside the so-called 'period gap' opens a new interesting problem about the continuity in the evolution of CVs.

The rare AM CVn stars have extremely short orbital periods, between 10 and 65 minutes, and their spectra show no evidence for hydrogen. They appear to be helium-rich versions of CVs. They are still waiting for a general model. They are probably binary systems of two white dwarfs, but even this is still controversial.

Despite all the work developed during the last decades, the problem of modeling accretion disks in CVs is by no means closed, especially in quiescence. Closely related is the problem of the cause of outbursts. We really do not know which of the present two families of models (Disk Instability Models or Secondary Instability Models) is responsible for the CVs outburst phenomenon, or in which system is each model valid, although Martinez-Pais et al. (1996) gave a contribution in solving this problem at least in the case of SS Cygni; they found some evidence for an increase of the mass transfer rate from the secondary star as the mechanism responsible for symmetric outbursts. Something similar can be said about the super-outburst phenomenon in SU UMa systems.

Gaudenzi et al. (1990), analyzing IUE spectra of SS Cygni, discussed about the outburst production as due to the destruction of the accretion disk. The matter slowly accretes onto the WD. Long and short outbursts correspond to total or partial destruction of the disk, respectively.

Alternatively, could nuclear burning be responsible of the production of outbursts in CVs? Indeed, nuclear burning onto white dwarf' surface was proposed by Mitrofanov $(1978,1980)$ as a mechanism suitable to generate X-rays in CVs. In spite of this shrewd suggestion, the community of theoreticians did not consider such a mechanism - certainly possible - worthy of taking up a part of their time. However, we believe that this alternative solution in explaining the generation of outbursts in CVs would deserve theoretician community's care. For instance, the white dwarf surface interested in the accretion in the system SS Cygni has been evaluated as $24 \%$ of the total (Gaudenzi et al., 2002). There, nuclear burning could occur.

Accretional heating by periodic DN events increases substantially the surface temperature of the WD in CVs (Godon \& Sion, 2002). Then, the envelope thermal structure resulting from compression and irradiation should be a crucial component in understanding the envelope structure of a pre-nova WD.

Another problem still open is connected with the classification of CVs in three kinds, namely NMCVs, PCVs and IPCVs. This is, in our opinion, another convenient classification, although artificial, probably not necessary if CVs are studied as gravimagnetic rotators. In this way a smooth evolution of the systems could be responsible of the variations of the gravimagnetic parameters. 
Are the IPCVs and PCVs smoothly connected via the SW Sex-like systems placed just in between? SW Sex systems have indeed orbital periods belong to the so-called 'period gap', and then their presence there sure cancel that gap.

Could some systems behave in different ways depending on their instantaneous physical conditions? For this reason they could apparently behave sometimes as PCVs and sometimes as NPCVs.

An example very clear is that of SS Cygni, usually classified as a non-magnetic dwarf nova. It has been detected by the INTEGRAL observatory in a region of the spectrum (up to $\sim 100$ $\mathrm{keV})$. This emission is very hard to be explained without the presence of polar caps in the WD of the system. Several proofs have been shown and discussed many times by Giovannelli's group in order to demonstrate the Intermediate Polar nature of it (e.g., Giovannelli, 1996, and references therein; Giovannelli \& Sabau-Graziati, 1998; 2012a); indeed, SS Cygni shows characteristics of a NMCV, as well as those of IP and sometimes even those of polars, although its position in the $\log \mathrm{P}_{\text {spin }}-\log \mathrm{P}_{\text {orb }}$ plane is very close to the line where IPs lie.

Important results are coming from the SPITZER space telescope with the detection of an excess (3-8) $\mu \mathrm{m}$ emission from MCVs, due to dust (Howell et al., 2006; Brinkworth et al., 2007). Gaudenzi et al. (2011) discussed about the reasons of the variable reddening in SS Cyg and demonstrated that this reddening is formed by two components: the first is interstellar in origin, and the second (intrinsic to the system itself) is variable and changes during the evolution of a quiescent phase. Moreover, an orbital modulation also exists. The physical and chemical parameters of the system are consistent with the possibility of formation of fullerenes.

The SPITZER space telescope detected the presence of fullerenes in a young planetary nebula (Cami et al., 2010). Fullerenes are the first bricks for the emergence of the life. Therefore, the possible presence of fullerenes in CVs opens a new line of investigation, foreboding of new interesting surprises.

Further information can be considered in order to better synthetize the open problems in the knowledge of CVs and related objects.

Sion (http://astronomy.villanova.edu/faculty/sion/CV/index.html) states that in the Galaxy we could expect $\approx 10^{6} \mathrm{CVs}$. One of the big questions that arises is: "can all of the observed CVs and the phenomena associated with them be understood in terms of a single unified picture?" Other questions relate to the relative probabilities that CVs will be observed at particular stages in their evolution, and how the observations of CVs at the current epoch can be used to determine their ultimate fate. To address these questions Nelson (2012) and Goliasch \& Nelson (2015) have undertaken a massive computational effort to theoretically simulate the evolution of most of the possible CVs that could be produced by nature. The temporal evolution of 56,000 nascent CVs was followed over an age of 10 billion years using the MESA stellar evolution code. According to Nelson, "This is the most ambitious analysis of the properties of an entire CV population that has ever been undertaken. The whole project required several core-years of CPU time."

While many of the results confirmed what had already been inferred about the properties of $\mathrm{CVs}$, there were a number of surprises including the identification of a number of previously unexplored evolutionary pathways. But, as expected, a sharp bifurcation was found between nascent $\mathrm{CVs}$ that evolved to produce double white-dwarf binaries (including ones containing helium and hybrid white dwarfs), and ones that continuously transferred mass over the lifetime of the universe. 
In addition, the predictions of the theoretical simulations were in good general agreement with the observations of CVs with reasonably well-measured properties.

What was surprising was the large number of short-period "ultracompact" binaries (AM CVn stars) that were produced and, especially, the enormous depletion of carbon relative to nitrogen and oxygen that is predicted at certain epochs for evolved systems. As Nelson points out, "It seems that nature has provided us with a unique way to identify CVs that descended from a highly evolved state based on their carbon abundances. There is already some observational evidence to suggest that there is a significant depletion of carbon in certain CVs. This could be a really critical test that will allow us to infer the lineage of some CVs and predict what their fate will be".

A recent paper by Otulakowska-Hypka, Olech \& Patterson (2016) present a statistical study of all measurable photometric features of a large sample of dwarf novae during their outbursts and superoutbursts. They used all accessible photometric data for all their objects to make the study as complete and up to date as possible. Their aim was to check correlations between these photometric features in order to constrain theoretical models which try to explain the nature of dwarf novae outbursts. They managed to confirm a few of the known correlations, that is the Stolz and Schoembs relation, the Bailey relation for long outbursts above the period gap, the relations between the cycle and supercycle lengths, amplitudes of normal and superoutbursts, amplitude and duration of superoutbursts, outburst duration and orbital period, outburst duration and mass ratio for short and normal outbursts, as well as the relation between the rise and decline rates of superoutbursts. However, they question the existence of the Kukarkin-Parenago relation but they found an analogous relation for superoutbursts. They also failed to find one presumed relation between outburst duration and mass ratio for superoutbursts. This study should help to direct theoretical work dedicated to dwarf novae.

Szkody \& Gänsicke (2012) provided a list of unanswered problems and questions and references for seeking additional information. Indeed, while the general evolutionary picture and the characteristics of the types of CVs are known at some level, there are major unsolved questions which remain. These include:

1. What is the actual number density and distribution of CVs in the Galaxy?

2. What happens to CVs once they reach the period minimum?

3. What are the detailed physics occurring in the common envelope?

4. What is the correct physics to describe viscosity in accretion disks?

5. What is the correct angular momentum prescription below the gap (besides gravitational radiation) that can account for the observed period minimum spike and the exact period distribution?

6. What causes the period gap?

7. How do Polars form and why are no magnetic white dwarfs in wide binaries observed? Are LARPS (Low Accretion Rate Polars) the progenitors of polars? Is there a difference in the emergence of systems containing magnetic white dwarfs versus non-magnetic? 
8. What causes Polars, as well as the novalike disk systems with orbital periods between 3 and 4 hours, to cease mass transfer and enter low states? Are the associated mass transfer variations of the companion stars a general phenomenon among all CVs?

9. Can the white dwarfs in CVs grow in mass?

10. Do CVs contain exoplanets?

I can add one point more:

11. Do CVs emit gravitational waves?

A possible answer to this point has been discussed by Poggiani (2017b), who reports that the most probable sources of GWs are AM CVn systems.

In order to answer to these not yet solved problems, a series of biennial Palermo Workshops about "The Golden Age of Cataclysmic Variables and Related Objects" has been organized since 2011. The refereed proceedings can be found in Giovannelli \& Sabau-Graziati (2012c, 2015c, 2017).

\section{Conclusions}

At the end of this review it appears evident the role of the magnetic field intensity at the surface of the white dwarf in CVs. It was also remarked the importance of studying the evolutionary path of CVs that very probably is a continuous path connecting the so-called NMCVs with MCVs.

Indeed, the detection of several SW Sex systems having orbital periods inside the so-called 'period gap' opens a new interesting problem about the continuity in the evolution of CVs. Are the IPCVs and PCVs smoothly connected via the SW Sex-like systems placed just in between?

In order to fully understand the emission properties and evolution of CVs, the mass-transfer process needs to be clearly understood, especially magnetic mass transfer, as well as the properties of magnetic viscosity in the accretion disks around compact objects. Consequently, the investigation on the magnetic field intensities in WDs appears crucial in understanding the evolution of CVs systems, by which it is possible to generate classical novae (e.g., Isern et al., 1997) and type-Ia supernovae (e.g., Isern et al., 1993).

In those catastrophic processes the production of light and heavy elements, and then the knowledge of their abundances provides strong direct inputs for cosmological models and cosmic ray generation problems.

We want to conclude with a general warning, apparently underestimated, like remarked by Giovannelli \& Sabau-Graziati (2015b): if we have not experimental information about the cross sections of nuclear reactions occurring in the stars it is hard to describe the correct star evolution.

The LUNA (Laboratory for Underground Nuclear Astrophysics) is devoted to measure nuclear cross sections relevant in astrophysics and astroparticle physics. It is the most valuable experiment running underground in the Gran Sasso Laboratory of the INFN (e.g. review by Broggini et al., 2010, 2016a,b, 2017). LUNA experiment provided the measures of the cross-sections of many nuclear reactions occurring in the stars for a better knowledge of stellar evolution (see Giovannelli \& Sabau-Graziati, 2015b, and references therein). 
A general data base for Experimental Nuclear Reaction Data (EXFOR) can be found in: https://www-nds.iaea.org/exfor/exfor.htm.

Acknowledgments This research has made use of NASA's Astrophysics Data System.

\section{References}

[1] Aldering, G.: 2005, NewAR 49, 346-353.

[2] Aleksic, J., Ansoldi, S., Antonelli, L.A., Antoranz, P., Babic, A. et al.: 2014, A\&A, 568, id. A109, 8 pp.

[3] Bath, G.T., van Paradijs, J.: 1983, Nature, 305, 33.

[4] Beskrovnaya, N.: 2017, talk at the Palermo Workshop 2017 on The Golden Age of Cataclysmic Variables and Related Objects - IV.

[5] Bhat, C.L., Kaul, R.K., Rawat, H.S., Senecha, V.K., Rannot, R.C., et al.: 1991, ApJ, 369, 475.

[6] Bianchini, A.: 1990, in Physics of Classical Novae, A. Cassatella \& R. Viotti (eds.), Springer-Verlag, Lecture Notes in Physics, 369, 13.

[7] Bianco, F.B., Howell, D.A., Sullivan, M., Conley, A., Kasen, D., et al.: 2011, ApJ, 741, 20.

[8] Bird, A.J., Malizia, A., Bazzano, A., Barlow, E.J., Bassani, L., et al.: 2007, ApJS, 170, 175.

[9] Bird, A.J., Bazzano, A., Bassani, L., Capitanio, F., Fiocchi, M., et al.: 2010, ApJS, $186,1$.

[10] Bisikalo, D.V., Kononov, D.A., Kaigorodov, P.V., Zhilkin, A.G., Boyarchuk, A.A.: 2008, ARep 52, 318-326.

[11] Bisikalo, D.V., Zhilkin, A.G.: 2012, in From Interacting Binaries to Exoplanets: Essential Modeling Tools, M.T. Richards \& I. Hubeny (Eds.), IAU Symp. 282, 509-516.

[12] Bisikalo, D.V., Kaygorodov, P.V., Ionov, D.E.: 2013, in Numerical Modeling of Space Plasma Flows (ASTRONUM2012), N.V. Pogorelov, E. Audit \& G.P. Zank (Eds.), ASP Conf. Ser. 474, 41-46.

[13] Bisikalo, D., Kaygorodov, P., Ionov, D., Shematovich, V., Lammer, H., Fossati, L.: 2013, ApJ 764, article id. 19, 5 pp.

[14] Bisikalo, D.V., Kurbatov, E.P., Pavlyuchenkov, Ya.N., Zhilkin, A.G., Kaygorodov, P.V.: 2016, MNRAS 458, 3892-3903.

[15] Bloom, J., Castro-Tirado, A.J., Hanlon, L., Kotani, T. (Eds.): 2010, I Workshop on Robotic Autonomous Observatories, Hindawi Publishing Corporation, Advances in Astronomy, Vol. 2010.

[16] Bode, M.F.: 2011a, arXiv: 0911.5254v3.

[17] Bode, M.F.: 2011b, arXiv: 111.4941v1.

[18] Bode, M.F., Evans, A. (eds.): 2008, Classical Novae, Cambridge Astrophys. Ser., No. 43, Cambridge University Press.

[19] Boneva, D., Kaigorodov, P.V., Bisikalo, D.V., Kononov, D.A.: 2009, ARep 53, 1004-1012.

[20] Brinkworth, C.S., Hoard, D.W., Wachter, S., Howell, S.B., Ciardi, D.R., et al: 2007, in 15th European Workshop on WDs, R. Napiwotzki \& M. R. Burleigh (eds.), ASP Conf. Ser. 372, 333.

[21] Broggini, C., Bemmerer, D., Guglielmetti, A., Menegazzo, R.: 2010, Annu. Rev. Nucl. Part. Sci. 60, 53-73. 
[22] Broggini, C.: 2016a, Journal of Physics: Conf. Ser. 703, Issue 1, article id. 012006.

[23] Broggini, C.: 2016b, in Frontier Research in Astrophysics II, online at https://pos.sissa.it/cgi-bin/reader/conf.cgi?confid=269, id.5

[24] Broggini, C.: 2017, in 6th Roma International Conference on Astroparticle Physics, Morselli, A., Capone, A., Rodriguez Fernandez, G. (Eds.), EPJ Web of Conferences, Volume 136, id.01008.

[25] Broggini, C., Bemmerer, D., Guglielmetti, A., Menegazzo, R.: 2010, Annu. Rev. Nucl. Part. Sci. 60, 53-73.

[26] Brown, W.R., Kilic, M., Allende Prieto, C.A., Kenyon, S.J.: 2011, MNRAS, 411, L31.

[27] Buckley, D.: 2015, talk at the Palermo Workshop on "The Golden Age of Cataclysmic Variables and Related Objects - III".

[28] Buckley, D.: 2017a, talk at the Palermo Workshop 2017 on The Golden Age of Cataclysmic Variables and Related Objects - IV.

[29] Buckley, D.: 2017b, talk at the Bursting Universe by Robots Eyes Workshop, Moscow, Lomonosov MSU, SAI.

[30] Buckley, D.A.H., Meintjes, P.J., Potter, S.B., Marsh, T.R., Gänsicke, B.T.: 2017, Nat. As. 1, id. 0029.

[31] Caballero-García, M.D., Pandey, S.B., Hiriart, D. \& Castro-Tirado, A.J. (Eds.): 2016, IV Workshop on Robotic Autonomous Observatories, RMxAC, Vol. 48.

[32] Cami, J., Bernard-Salas, J., Peeters, E., Malek, S.E.: 2010, Sci., 329, 1180.

[33] Cassatella, A., Viotti, R. (eds.): 1990, Physics of Classical Novae, Springer-Verlag, Lecture Notes in Physics, 369.

[34] Castro Cerón, J.M.: 2011, talk at the Frascati Workshop 2011 on "Multifrequency Behaviour of High Energy Cosmic Sources".

[35] Castro-Tirado, A.J.: 2008, in 3rd Symposium of the Astrophysics Group of the Spanish Royal Physical Society (RSEF), A. Ulla \& M. Manteiga (Eds.), Lecture Notes and Essays in Astrophysics 3, 131.

[36] Castro-Tirado, A.J.: 2010a, Adv. Astron. Vol. 2010, Article ID 570489, 8 pages.

[37] Castro-Tirado, A.J.: 2010b, Adv. Astron. Vol. 2010, Article ID 824731, 1 page.

[38] Chadwick, P.M., Dickinson, J.E., Dickinson, M.R., Dipper, N.A., Holder, J. et al.: 1995, APh, 4, 99.

[39] Chiosi, E., Chiosi, C., Trevisan, P., Piovan, L., Orio, M.: 2015, MNRAS 448, 2100.

[40] Churazov, E., Sunyaev, R., Isern, J., Knödlseder, J., Jean, P. et al.: 2014, Nature 512, 406.

[41] Churazov, E., Sunyaev, R., Isern, J., Bikmaev, I., Bravo, E. et al.: 2015, ApJ 812, 62.

[42] Cooley, J.W., Tukey, J.W.: 1965, An algorithm for the machine calculation of complex Fourier series, Math. Comput. 19, 297-301.

[43] Darnley, M.J., Ribeiro, V.A.R.M., Bode, M.F., Hounsell, R.A., Williams, R.P.: 2011, arXiv: $1112.2589 \mathrm{v} 1$.

[44] Darnley, M.J., Bode, M.F., Harman, D.J., Hounsell, R.A., Munari, U. et al.: 2014, in Stella Novae: Past and Future Decades, P.A. Woudt \& V.A.R.M. Ribeiro (Eds.). ASP Conf. Ser. Vol. 490, 49.

[45] De Angelis, A., Tatischeff, V., Tavani, M., Oberlack, U., Grenier, I., et al.: 2017, Exp. Astron. 44, Issue $1,25-82$. 
[46] Evans, A.: 2011, in Asymmetric Planetary Nebulae 5 Conference, A.A. Zijlstra, F. Lykou, I. McDonald, \& E. Lagadec (eds.), Jodrell Bank Centre for Astrophysics, p. 305.

[47] Evans, A., Bode, M.F., O’Brien, T.J., Darnley, M.J. (eds.): 2008, RS Ophiuchi (2006) and the Recurrent Nova Phenomenon, ASP Conf. Ser., Vol. 401.

[48] Fabbiano, G., Hartmann, L., Raymond, J. Steiner, J., Branduardi-Raymond, G., Matilsky, T.: 1981, ApJ, 243, 911.

[49] Fabian, A.C.: 1988, Origins. The Darwin College lectures (1986), Fabian, A.C. (Ed.), Cambridge University Press.

[50] Ferrario, L., Wickramasinghe, D.T.: 2005, MNRAS 356, 615-620.

[51] Ferrario, L., Wickramasinghe, D.T.: 2007, ASP Conf. Ser. 372, 163-168.

[52] Ferrario, L., de Martino, D., Gänsicke, B.T.: 2015, SSRv 191, 111-169.

[53] Ferrario, L., Melatos, A., Zrake, J.: 2015, SSRv 191, 77-109.

[54] Gänsicke, B.T.: 2005, in The Astrophysics of Cataclysmic Variables and Related Objects, J.-M. Hameury \& J.-P. Lasota (Eds.), ASP Conf. Ser. Vol. 330, 3.

[55] Gaudenzi, S., Giovannelli, F., Lombardi, R., Claudi, R.: 1986, in New Insights in Astrophysics, E. Rolfe (ed.), ESA SP-263, 455.

[56] Gaudenzi, S., Giovannelli, F., Lombardi, R., Claudi, R.: 1990, AcA, 40, 105

[57] Gaudenzi, S., Claudi, R.U., Giovannelli, F., Lombardi, R., Pelosi, M., Strappolini, M.: 2002, in Multifrequency Behaviour of High Energy Cosmic Sources, F. Giovannelli \& L. Sabau-Graziati (eds.), Mem. S.A.It., 73 N. 1, 213.

[58] Gaudenzi S., Giovannelli, F., Mandalari, M., Corradini, M., Lombardi, R.: 2011, A\&A 525, 147.

[59] Geng, J-J., Zhang, B., Huang, Y.-F.: 2016, ApJL 831, Issue 1, article id. L10, 5 pp.

[60] Gilmozzi, R., Spyromilio, J.: 2007, The Messenger 127, 11-19.

[61] Giovannelli, F. (Ed.): 1985 Multifrequency Behaviour of Accreting Galactic Sources, SIDEREA, Roma, pp. 371.

[62] Giovannelli, F.: 1996, in Multifrequency Behaviour of High Energy Cosmic Sources, F. Giovannelli \& L. Sabau-Graziati (eds.), Mem. S.A.It., 67, 401.

[63] Giovannelli, F. (Ed.): 2001a, The Bridge between the Big Bang and Biology (Stars, Planetary Systems, Atmospheres, Volcanoes: Their Link to Life), President Bureau of the CNR, Roma, Italy, pp. 1-440.

[64] Giovannelli, F.: 2016, in 4th Annual Conference on High Energy Astrophysics in Southern Africa, online at http://pos.sissa.it/cgi-bin/reader/conf.cgi?confid=275, id.31.

[65] Giovannelli, F., Sabau-Graziati, L.: 1998, in Ultraviolet Astrophysics Beyond the IUE Final Archive, W. Wamstecker \& R.Gonzalez-Riestra (eds.), ESA SP-413, 419.

[66] Giovannelli, F., Sabau-Graziati, L.: 2012a, in The Golden Age of Cataclysmic Variables and Related Objects, F. Giovannelli \& L. Sabau-Graziati (eds.), Mem. S.A.It., 83 N. 2, 698.

[67] Giovannelli, F., Sabau-Graziati, L.: 2012b, in Second Workshop on Robotic Autonomous Observatories, S. Guziy, S.B. Pandey, J.C. Tello \& A.J. Castro-Tirado (Eds.), Astronomical Society of India Conf. Ser. 7, 59-78. 
[68] Giovannelli, F., Sabau Graziati, L.: 2015a, in The Golden Age of Cataclysmic Variables and Related Objects - III, online at http://pos.sissa.it/cgi-bin/reader/conf.cgi?confid=246, id.1.

[69] Giovannelli, F., Sabau Graziati, L.: 2015b, in Multifrequency Behaviour of High Energy Cosmic Sources - XI, online at http://pos.sissa.it/cgi-bin/reader/conf.cgi?confid=246, id.1.

[70] Giovannelli, F., Sabau-Graziati, L.: 2016a, in Accretion Processes in Cosmic Sources, online at http://pos.sissa.it/cgi-bin/reader/conf.cgi?confid=288, id.1.

[71] Giovannelli, F., Sabau-Graziati, L. (Eds.): 2016b, Accretion Processes in Cosmic Sources: Young Stellar Objects, Cataclysmic Variables and Related Objects, X-ray Binary Systems, Active Galactic Nuclei, Online at http://pos.sissa.it/cgi-bin/reader/conf.cgi?confid=288.

[72] Giovannelli, F., Sabau-Graziati, L.: 2016c, in IV Workshop on Robotic Autonomous Observatories, María Dolores Caballero-García, Shasi B. Pandey, David Hiriart \& Alberto J. Castro-Tirado (Eds.), RMxAC 48, 1-15.

[73] Giovannelli, F., Sabau-Graziati, L.: 2017, in Multifrequency Behaviour of High Energy Cosmic Sources - XII, online at https://pos.sissa.it/cgi-bin/reader/conf.cgi?confid=306, id.1.

[74] Giovannelli, F., Sabau-Graziati, L.: 2018, in The AstroRob 2017 Proceedings, Rev. Mex AA (in press).

[75] Godon, P., Sion, E.M.: 2002, ApJ, 566, 1084.

[76] Goliasch, J., Nelson, L.: 2015, ApJ 809, 80.

[77] Greggio, L., Renzini, A., Daddi, E.: 2008, arXiv: 0805.1512v2.

[78] Guy, J., Sullivan, M., Conley, A., Regnault, N., Astier, P., et al.: 2010, A\&A, 523, 7.

[79] Guziy, S., Pandey, S.B., Tello, J.C. \& Castro-Tirado, A.J. (Eds.): 2012, II Workshop on Robotic Autonomous Observatories, Astron. Soc. of India Conf. Ser., Vol. 7.

[80] Hack, M., la Dous, C.: 1993, in Cataclysmic Variables and Related Objects, M. Hack \& C. la Dous (eds.), NASA SP-507, 1.

[81] Hamuy, M., Pignata, G., Maza, J., Clocchiatti, A., Anderson, J., et al.: 2012, Mem. S.A.It. Vol. 83, 388-392.

[82] Harrison, T.E., McNamara, B.J., Szkody, P., McArthur, B.E., Benedict, G.F. et al.: 1999, ApJL 515, L93.

[83] Hellier, C.: 2001, Cataclysmic Variable Stars, Springer

[84] Hessman, F.V.:: 2001a, in Small Telescope Astronomy on Global Scales, Bohdan Paczynski, Wen-Ping Chen, and Claudia Lemme (Eds.), ASP Conf. Ser. 246, 13.

[85] Hessman, F.V.:: 2001b, in Small Telescope Astronomy on Global Scales, Bohdan Paczynski, Wen-Ping Chen, and Claudia Lemme (Eds.), ASP Conf. Ser. 246, 357.

[86] Hillas, A.M.: 1984, ARA\&A 22, 425-444.

[87] Horne, K.: 1985, MNRAS 213, 129-141.

[88] Howell, D.A., Conley, A., Della Valle, M., Nugent, P., Perlmutter, S., et al.: 2009, arXiv0903, 1086 H.

[89] Howell, S.B., Cash, J., Mason, K.O., Herzog, A.E.: 1999, AJ 117, 1014.

[90] Howell, S.B., Brinkworth, C., Hoard, D.W., Wachter, S., Harrison, T., et al.: 2006, ApJL 646, L65. 
[91] Hoyle, F., Fowler, W.A.: 1960, ApJ, 132, 565.

[92] Hudec, R.: 2015, in The Golden Age of Cataclysmic Variables and Related Objects - III, online at http://pos.sissa.it/cgi-bin/reader/conf.cgi?confid=255, id.41.

[93] Hudec, R.: 2017, in Multifrequency Behaviour of High Energy Cosmic Sources -XII, online at https://pos.sissa.it/cgi-bin/reader/conf.cgi?confid=306, id.83

[94] Hudec, R., Hudec, L., Klíma, M.: 2012, Acta Polytechnica, 52, No. 1, 27.

[95] Hudec, R., Hudec, L.: 2013, Acta Polytechnica, 53, No. 3, 23.

[96] Iben, I., Jr., Tutukov, A.V.: 1984, ApJS, 54, 335.

[97] Ikhsanov, N.: 2017, talk at the Palermo Workshop 2017 on The Golden Age of Cataclysmic Variables and Related Objects - IV.

[98] Illarionov, A.F., Sunyaev, R.A.: 1975, A\&A 39, 185.

[99] Isakova, P.: 2017, talk at the Palermo Workshop 2017 on The Golden Age of Cataclysmic Variables and Related Objects - IV.

[100] Isern, J., Hernanz, M., García-Berro, E.: 1993, in White Dwarfs: Advances in Observation and Theory, M.A. Barstow (ed.), Kluwer Academic Publ., Dordrecht, Holland, NATO ASI Ser., C403, 139.

[101] Isern, J., Hernanz, M., Abia, C., José, J.: 1997, in Frontier Objects in Astrophysics and Particle Physics, F. Giovannelli \& G. Mannocchi (eds.), Italian Physical Society, Editrice Compositori, Bologna, Italy, 57, 113.

[102] Katz, J.I.: 2017, ApJ 835, Issue 2, article id. 150, 4 pp.

[103] Keating, Brian G. et al.: 2003, SPIE 4843, 284.

[104] Kilic, M., Brown, W.R., Allende Prieto, C., Agüeros, M.A.; Heinke, C., Kenyon, S.J.: 2011, ApJ, $727,3$.

[105] Kistler, M.D., Stanek, K.Z., Kochanek, C.S., Prieto, J.L., Thompson, T.A.: 2013, ApJ, $770,88$.

[106] Knigge, C.: 2006, MNRAS, 373, 484.

[107] Knigge, C.: 2010, AIPC, 1314, 171.

[108] Knigge, C.: 2011, ASPC, 447, 3.

[109] Knigge, C., Baraffe, I., Patterson, J.: 2011, ApJS, 194, 28.

[110] Kononov, D.A., Giovannelli, F., Bruni, I., Bisikalo, D.V.: 2012, A\&A 538, id. A94, 7 pp.

[111] Körding, E., Rupen, M., Knigge, C., Fender, R., Dhawan, V., et al.: 2008, Science 320, 1318.

[112] Kotze, E.J., Potter, S.B., McBride, V.A.: 2015, A\&A 579, id. A77, 9 pp.

[113] Kotze, E.J., Potter, S.B., McBride, V.A.: 2016, A\&A 595, id. A47, 12 pp.

[114] Kowalski, M., Rubin, D., Aldering, G, Agostinho, R.J., Amadon, A., et al.: 2008, ApJ, 686, 749.

[115] Landi, R., Bassani, L., Dean, A.J., Bird, A.J., Fiocchi, M. et al.: 2009, MNRAS 392, 630.

[116] Lang, M.J., Buckley, J.H., Carter-Lewis, D.A., Catanese, M., Cawley, M.F. et al.: 1998, APh, 9, 203.

[117] Levitan, D.: 2013, Finding Needles in the Haystack: A Search for AM CVn Systems using the Palomar Transient Factory, Ph.D. Thesis, California Institute of Technology, U.S.A. 
[118] Levitan, D., Groot, P.J., Prince, Th.A., Kulkarni, S.R., Laher, R. et al.: 2015, MNRAS 446, 391.

[119] Lipunov, V.M.: 1987, Ap\&SS 132, no. 1, 1-51.

[120] Lipunov, V.M., Postnov, K.A.: 1988, Ap\&SS 145, no. 1, 1-45.

[121] Lipunov, V. M., Nazin, S.N., Panchenko, I.E., Postnov, K.A., Prokhorov, M.E.: 1995, A\&A 298, 677-687.

[122] Lipunov, V. et al.: 2010, Advances in Astronomy, 2010, article id. 349171.

[123] Lira, P.: 1995, Masters thesis, Univ. Chile.

[124] Luisi, P.L., Capra, F.: 2014, The systems View of Life, Cambridge Univ. Press, 510 pp.

[125] Maguire, K., Sullivan, M., Ellis, R.S., Nugent, P.E., Howell, D.A. et al.: 2012, MNRAS, 426, 2359.

[126] Mannucci, F., Della Valle, M., Panagia, N.: 2006, MNRAS, 370, 773.

[127] Marsh, T.R.: 2001, in Astrotomography, Indirect Imaging Methods in Observational Astronomy, H.M.J. Boffin, D. Steeghs \& J. Cuypers (Eds.), Lecture Notes in Physics 573, 1-27.

[128] Marsh, T.R., Horne, K.: 1988, MNRAS 235, 269-286.

[129] Marsh, T.R., Gänsicke, B.T., Hümmerich, S., Hambsch, F.-J., Bernhard, K., et al.: 2016, Nature 537, Issue 7620, 374-377.

[130] Martinez-Pais, I.G., Giovannelli, F., Gaudenzi, S, Rossi, C.: 1996, A\&A, 308, 833.

[131] Meintjes, P.: 2017, talk at the Palermo Workshop 2017 on The Golden Age of Cataclysmic Variables and Related Objects - IV.

[132] Meintjes, P.J., Raubenheimer, B.C., de Jager, O.C., Brink, C., Nel, H.I., et al.: 1992, ApJ, 401, 325.

[133] Meintjes, P.J., Oruru, B., Odendaal, A.: 2012, in The Golden Age of Cataclysmic Variables and Related Objects, F. Giovannelli \& L. Sabau-Graziati (eds.), Mem SAIt., 83, 643.

[134] Mikołajewska, J.: 2011, in Binary Paths in Type Ia Supernovae Explosions, R. Di Stefano \& M. Orio (eds.), IAU Symp. No. 281 (in press). 2011, arXiv: 1110.1847v1.

[135] Mitrofanov, I.G.: 1978, Sov. Astron. Lett., 4, 119.

[136] Mitrofanov, I.G.: 1980, in Close Binary Stars: Observations and Interpretations, D.M. Popper \& R.K. Ulrich (eds.), D. Reidel Publ. Co., Dordrecht, Holland, p. 431.

[137] Nelson, L.: 2012, J. Phys.: Conf. Ser. Volume 341, Issue 1, id. 012008.

[138] Ogburn, R.W., IV et al.: 2010, SPIE 7741E..1GO.

[139] Otulakowska-Hypka, M., Olech, A., Patterson, J.: 2016, MNRAS 460, Issue 3, 2526-2541.

[140] Patat, F., Chandra, P., Chevalier, R., Justham, S., Podsiadlowski, Ph., et al.: 2008, The Messenger, $131,30$.

[141] Patterson, J.: 1992, ApJ 384, 234.

[142] Patterson, J.: 2014, in 33rd Annual Symposium on Telescope Science, Society for Astron. Sci., 33, $17-22$.

[143] Patterson, J., Uthas, H., Kemp, J., de Miguel, E., Krajci, Th.: 2013, MNRAS, 434, 1902.

[144] Pekurovsky, D.: 2012, Siam J. Sci. Comput. Vol. 34, No. 4, pp. C192ŰC209, Society for Industrial and Applied Mathematics. 
[145] Phillips, M.M.: 2005, ASPC, 342, 211.

[146] Phillips, M.M.: 2011, arXiv: 11.4463v1.

[147] Phillips, M.M., Lira, P., Hamuy, M., Maza, J.: 1999, AJ, 118, 1766.

[148] Pignata, G., Maza, J., Antezana, R., Cartier, R., Folatelli, G., et al.: 2009, AIPC, 1111, 551-554.

[149] Poggiani, R.: 2017a, in Multifrequency Behaviour of High Energy Cosmic Sources - XII, online at https://pos.sissa.it/cgi-bin/reader/conf.cgi?confid=306, id.53.

[150] Poggiani, R.: 2017b, in The Golden Age of Cataclysmic Variables and Related Objects - IV, in press.

[151] Rappaport, S., Verbunt, F., Joss, P.C.: 1983, ApJ, 275, 713.

[152] Rees, M.J.: 1988, in Origins, A.C. Fabian (ed.), Cambridge University Press, 1.

[153] Ritter, H.: 1992, in The Astronomy and Astrophysics Encyclopedia, Cambridge University Press, Cambridge, UK, p. 61.

[154] Rix, H-W., Bovy, J.: 2013, A\&A Rev. 21, article id. 61.

[155] Rodriguez-Gil, P.: 2003, Ph.D. Thesis, La Laguna University, Spain.

[156] Salewski, M., Geiger, B., Heidbrink, W.W., Jacobsen, A.S., S B Korsholm, S.B., et al. (the ASDEX Upgrade Team): 2015, Plasma Phys. Control. Fusion 57, 014021, 10 pp.

[157] Scalzo, R.A., Ruiter, A.J., Sim, S.A.: 2014, MNRAS, 445, 2535.

[158] Scaringi, S.: 2015, talk at the Palermo Workshop on The Golden Age of CVs and Related Objects III.

[159] Scaringi, S., Bird, A.J., Norton, A.J., Knigge, C., Hill, A.B. et al.: 2010, MNRAS 401, 2207.

[160] Schaefer, B.E.: 2010, ApJS, 187, 275.

[161] Schaefer, B.E.: 2011, ApJ, 742, 112.

[162] Schmidtobreick, L.: 2013, Central European Astrophysical Bulletin 37, 361-368.

[163] Schmidtobreick, L., Tappert, C.: 2014, in Stella Novae: Past and Future Decades, P.A. Woudt \& V.A.R.M. Ribeiro (Eds.). ASP Conf. Ser. Vol. 490, 29.

[164] Schmidtobreick, L., Tappert, C.: 2015, Acta Polytechnica CTU Proc. Vol. 2, 188.

[165] Seitter, W.C..: 1990, in Physics of Classical Novae, A. Cassatella \& R. Viotti (eds.), Springer-Verlag, Lecture Notes in Physics, 369, 79.

[166] Shafter, A.W., Curtin, C., Pritchet, C.J., Bode, M.F., Darnley, M.J.: 2014, in Stella Novae: Past and Future Decades, P.A. Woudt \& V.A.R.M. Ribeiro (eds.), ASP Conf. Ser., 490, 77.

[167] Shafter, A.W., Henze, M., Rector, T.A., Schweizer, F., Hornoch, K. et al.: 2015, ApJS, 216, 34.

[168] Shandarin, S., Habib, S., Heitmann, K.: 2010, PhRv D, 81, Issue 10, id. 103006.

[169] Shimokawabe, T., Kawai, N., Mori, Y.A., Kudo, Y., Nakajima, H., et al.: 2009, AIPC 1133, $79-81$.

[170] Sidro, N., Cortina, J., Mauche, C.W., de Oña, E., Torres, D.F.: 2008, ICRC. 2, 715.

[171] Smak, J.: 1967, AcA, 17, 3.

[172] Smak, J.: 1985, in Multifrequency Behaviour of Galactic Accreting Sources, F. Giovannelli (ed.), SIDEREA, Roma, Italy, p. 3. 
[173] Stril, A., Cahn, R.N., Linder, E.V.: 2010, MNRAS, 404, 239.

[174] Surina, F., Bode, M.F., Darnley, M.J: 2011, arXiv: 1111.5524v1.

[175] Surina, F., Bode, M.F., Darnley, M.J.: 2015, PKAS, 30, 237.

[176] Szkody, P., Gänsicke, B.T.: 2012, JAAVSO 40, 563.

[177] Takata, J., Yang, H., Cheng, K.S.: 2017, ApJ 851, Issue 2, article id. 143, 13 pp.

[178] Tello, J.C., Riva, A., Hiriart, D. \& Castro-Tirado, A.J. (Eds.): 2014, III Workshop on Robotic Autonomous Observatories, RMxAC, Vol. 45.

[179] Terada, Y., Hayashi, T., Ishida, M., Mukai, K., Dotani, T. et al.: 2008, in High Energy Gamma-Ray Astronomy, AIP Conf. Proc. 1085, 689-692.

[180] Terada, Y., Ishida, M., Bamba, A., Mukai, K., Hayashi, T., Harayama, A.: 2010, ApJ, 721, 1908.

[181] Topchiev, N.P. et al.: 2016a, J. Ph. Conf. Ser. 675, 032009.

[182] Topchiev, N.P. et al.: 2016b, J. Ph. Conf. Ser. 675, 032010.

[183] Topchiev, N.P. et al.: 2017, J. Ph. Conf. Ser. 798, 012011.

[184] Townsley, D.M., Bildsten, L.: 2005, ApJ, 628, 395.

[185] Tsebrenko, D., Soker, N.: 2015, MNRAS, 447, 2568.

[186] Tsuru, T. Go: 2008, talk at the Vulcano Workshop on Frontier Objects in Astrophysics and Particle Physics.

[187] Tsuru, T. Go: 2009, in Frontier Objects in Astrophysics and Particle Physics, F. Giovannelli \& G. Mannocchi (Eds.), SIF, Editrice Compositori, Bologna, Italy, Vol. 98, 195-204.

[188] Voss, R., Nelemans, G.: 2008, Nature, 451, 802.

[189] Vrielmann, S., Cropper, M. (eds.): 2004, in Magnetic Cataclysmic Variables, IAU Colloquium 190, ASP Conf. Proc. Vol. 315. San Francisco: Astronomical Society of the Pacific.

[190] Warner, B.: 2002, in Classical Nova Explosions, AIP Conf. Proc., 637, 3.

[191] Webbink, R.F.: 1984, ApJ, 277, 355.

[192] Whelan, J., Iben, I. Jr.: 1973, ApJ, 186, 1007.

[193] Wickramasinghe, D.T., Ferrario, L.: 2000, PASP 112, Issue 773, 873-924.

[194] Wickramasinghe, D.T., Tout, C.A., Ferrario, L.: 2014, MNRAS 437, 675-681.

[195] Williams, S.C., Darnley, M.J., Bode, M.F., Shafter, A.W.: 2014, in Stella Novae: Past and Future Decades, P.A. Woudt \& V.A.R.M. Ribeiro (eds.), ASP Conf. Ser., 490, 85.

[196] Williams, S.C., Darnley, M.J., Bode, M.F., Shafter, A.W.: 2016, ApJ. 817. 143.

[197] Yaron, O., Prialnik, D., Shara, M.M., Kovetz, A.: 2005, ApJ, 623, 398.

[198] Yoon, S.-C., Podsiadlowski, Ph., Rosswog, S.: 2007, MNRAS, 380, 933.

[199] Zhilkin, A.G., Bisikalo, D.V.: 2009, in Numerical Modeling of Space Plasma Flows: ASTRONUM-2008, Nikolai V. Pogorelov, Edouard Audit, Phillip Colella \& Gary P. Zank (Eds.), ASP Conf. Ser. 406, 118-123.

[200] Zhilkin, A.G., Bisikalo, D.V.: 2011, in 5th international conference of numerical modeling of space plasma flows (astronum 2010), Nikolai V. Pogorelov, Edouard Audit \& Gary P. Zank, (Eds.), ASP Conf. Ser. 444, 91-96.

[201] Zhilkin, A.G., Bisikalo, D.V., Mason, P.A.: 2012, ARep 56, 257-274. 